\title{
Mathematical modeling of micropolar fluid flows through a thin porous medium
}

\author{
Francisco Javier SUÁREZ-GRAU
}

\begin{abstract}
We study the flow of a micropolar fluid in a thin domain with microstructure, i.e. a thin domain with thickness $\varepsilon$ which is perforated by periodically distributed solid cylinders of size $a_{\varepsilon}$. A main feature of this study is the dependence of the characteristic length of the micropolar fluid on the small parameters describing the geometry of the thin porous medium under consideration. Depending on the ratio of $a_{\varepsilon}$ with respect to $\varepsilon$, we derive three different generalized Darcy equations where the interaction between the velocity and the microrotation fields is preserved.
\end{abstract}

AMS classification numbers: 76A05, 76A20, 76M50, 76S05, 35B27, 35Q35.

Keywords: Homogenization; micropolar fluid flow; Darcy's law; thin-film fluid; thin porous medium.

\section{Introduction}

Based on the micropolar fluid theory [12, 13, which takes into account the effects of solid particles additive in a Newtonian fluid, we study flows of micropolar fluids in a thin domain which is perforated by periodically distributed solid cylinders (microstructure) which is called thin porous medium (TPM). This type of domains include two small parameters: one called $\varepsilon$ is connected to the fluid film thickness and the other called $a_{\varepsilon}$ to the microstructure representing the size of the cylinders and the interspatial distance between them. The behavior of fluid flows through TPM has been studied extensively, mainly because of its importance in many industrial processes, see 15, 16, 18, 19, 20, 21. However, the literature on non-Newtonian micropolar fluid flows in this type of domains is far less complete, although these problems have now become of great practical relevance. Therefore, the objetive of this paper is to derive generalized micropolar Darcy equations for the pressure depending on the magnitude of the parameters involving the TMP.

For Newtonian fluids, this problem has been addressed in 14 proving the existence of three types of TPM:

- The proportionally thin porous medium (PTPM), corresponding to the critical case when the cylinder height is proportional to the interspatial distance, with $\lambda$ the proportionality constant, that is $a_{\varepsilon} \approx \varepsilon$, with $a_{\varepsilon} / \varepsilon \rightarrow \lambda, 0<\lambda<+\infty$.

- The homogeneously thin porous medium (HTPM), corresponding to the case when the cylinder height is much larger than interspatial distance, i.e. $a_{\varepsilon} \ll \varepsilon$ which is equivalent to $\lambda=0$.

- The very thin porous medium (VTPM), corresponding to the case when the cylinder height is much smaller than the interspatial distance, i.e. $a_{\varepsilon} \gg \varepsilon$ which is equivalent to $\lambda=+\infty$.

In particular, starting from the Stokes system with body forces $f$, it can be deduced that the flow is governed by a 2D Darcy equation for the pressure $p$ of the form

$$
\operatorname{div}\left(K_{\lambda}(f-\nabla p)\right)=0 \quad(0 \leq \lambda \leq+\infty),
$$

*Departamento de Ecuaciones Diferenciales y Análisis Numérico. Facultad de Matemáticas. Universidad de Sevilla. 41012-Sevilla (Spain) fjsgrau@us.es 
where $K_{\lambda} \in \mathbb{R}^{2 \times 2}$ is a macroscopic quantity known as flow factor which takes into account the microstructure of the TPM. Moreover, it holds that the flow factor in the PTPM is calculated by solving 3D Stokes local problems depending on the parameter $\lambda$, while it is obtained in HTPM by solving 2D Stokes local problems and by solving 2D local Hele-Shaw problems in the VTPM, which it represents a considerable simplification.

These results were proved in 14 by using the multiscale expansion method, which is a formal but powerful tool to analyze homogenization problems, and later rigorously developed in [5] by using an adaptation of the periodic unfolding method 6, 9, 10. This adaptation consists of a combination of the unfolding method in the horizontal variables with a rescaling in the height variable, in order to work with a domain of fixed height, and then to use suitable compactness results to pass to the limit when the geometrical parameters $\varepsilon$ and $a_{\varepsilon}$ tend to zero. We remark that this adaptation was developed in [4] to study the case of non-Newtonian power law fluids in the TPM and it was recently applied to the case of non-Newtonian Bingham fluids in [2, 3].

Due to its importance in industrial and engineering applications, we consider a non-Newtonian micropolar fluid flow in TPM governed by the linearized micropolar equations with body forces $f$ and body torque $g$. By using the homogenization techniques developed in [4, we derive that the flow is governed by a generalized 2D Darcy equation for the pressure $p$ of the form

$$
\operatorname{div}\left(K_{\lambda}^{(1)}(f-\nabla p)+K_{\lambda}^{(2)} g\right)=0 \quad(0 \leq \lambda \leq+\infty)
$$

where the flow factors $K^{(k)} \in \mathbb{R}^{2 \times 2}, k=1,2$, are calculated depending on the cases as follows: by solving 3D micropolar local problems depending on the parameter $\lambda$ in the PTPM, by solving 2D micropolar local problems in the HTPM and by solving 2D local micropolar Reynolds problems in the case VTPM.

As far as the author knows, this is the first attempt to carry out such an analysis for micropolar fluids, which could be instrumental for understanding the effects on the flows of micropolar fluids and the microstructure of the domain. In view of that, more efficient numerical algorithms could be developed improving, hopefully, the known engineering practice.

The paper is organized as follows. In Section 2, we introduce some useful notation and in Section 3 , we give some a priori estimates for the dilated velocity, the microrotation and the pressure, we introduce the extension of the unknowns to the whole domain $\Omega$, and finally we recall the version of the unfolding method necessary to pass to the limit in the next sections. Namely, we analyze the case PTPM in Section 4 , the case HTPM in Section 5 and the case VTPM in Section 6 .

\section{Statement of the problem}

In this section, we first define the TPM and some sets necessary to study the asymptotic behavior of the solutions. Next, we introduce the problem considered and also the rescaled problem posed in a domain of fixed height. We finish this section giving the equivalent weak variational formulations.

Definition of the TPM. A periodic porous medium is defined by a domain $\omega$ and an associated microstructure, or periodic cell $Y^{\prime}=(-1 / 2,1 / 2)^{2}$ which is made of two complementary parts: the fluid part $Y_{f}^{\prime}$, and the solid part $Y_{s}^{\prime}\left(Y_{f}^{\prime} \cup Y_{s}^{\prime}=Y^{\prime}\right.$ and $\left.Y_{f}^{\prime} \cap Y_{s}^{\prime}=\emptyset\right)$. More precisely, we assume that $\omega$ is a smooth, bounded, connected set in $\mathbb{R}^{2}$ and that $Y_{s}^{\prime}$ is an open connected subset of $Y^{\prime}$ with a smooth boundary $\partial Y_{s}^{\prime}$, such that $\overline{Y_{s}^{\prime}}$ is strictly included in $Y^{\prime}$.

The microscale of a porous medium is given by a small positive number $a_{\varepsilon}$. The domain $\omega$ is covered by a regular mesh of size $a_{\varepsilon}$ : for $k^{\prime} \in \mathbb{Z}^{2}$, each cell $Y_{k^{\prime}, a_{\varepsilon}}^{\prime}=a_{\varepsilon} k^{\prime}+a_{\varepsilon} Y^{\prime}$ is divided in a fluid part $Y_{f_{k^{\prime}}, a_{\varepsilon}}^{\prime}$ and a solid part $Y_{s_{k^{\prime}}, a_{\varepsilon}}^{\prime}$, i.e. is similar to the unit cell $Y^{\prime}$ rescaled to size $a_{\varepsilon}$. We define $Y=Y^{\prime} \times(0,1) \subset \mathbb{R}^{3}$, which is divided in a fluid part $Y_{f}$ and a solid part $Y_{s}$, and consequently $Y_{k^{\prime}, a_{\varepsilon}}=Y_{k^{\prime}, a_{\varepsilon}}^{\prime} \times(0,1) \subset \mathbb{R}^{3}$, which is also divided in a fluid part $Y_{f_{k^{\prime}}, a_{\varepsilon}}$, and a solid part $Y_{s_{k^{\prime}}, a_{\varepsilon}}$.

We denote by $\tau\left(\bar{Y}_{s_{k^{\prime}}, a_{\varepsilon}}^{\prime}\right)$ the set of all translated images of $\bar{Y}_{s_{k^{\prime}}, a_{\varepsilon}}^{\prime}$. The set $\tau\left(\bar{Y}_{s_{k^{\prime}}, a_{\varepsilon}}^{\prime}\right)$ represents the solids in $\mathbb{R}^{2}$. The fluid part of the bottom $\omega_{\varepsilon} \subset \mathbb{R}^{2}$ of the porous medium is defined by $\omega_{\varepsilon}=\omega \backslash \bigcup_{k^{\prime} \in \mathcal{K}_{\varepsilon}} \bar{Y}_{s_{k^{\prime}}, a_{\varepsilon}}$, where 
$\mathcal{K}_{\varepsilon}=\left\{k^{\prime} \in \mathbb{Z}^{2}: Y_{k^{\prime}, a_{\varepsilon}}^{\prime} \cap \omega \neq \emptyset\right\}$. The whole fluid part $\Omega_{\varepsilon} \subset \mathbb{R}^{3}$ is defined by

$$
\Omega_{\varepsilon}=\left\{\left(x_{1}, x_{2}, x_{3}\right) \in \omega_{\varepsilon} \times \mathbb{R}: 0<x_{3}<\varepsilon\right\} .
$$

We make the assumption that the solids $\tau\left(\bar{Y}_{s_{k^{\prime}}, a_{\varepsilon}}^{\prime}\right)$ do not intersect the boundary $\partial \omega$. We define $Y_{s_{k^{\prime}}}^{\varepsilon}, a_{\varepsilon}=$ $Y_{s_{k^{\prime}}, a_{\varepsilon}}^{\prime} \times(0, \varepsilon)$. Denote by $S_{\varepsilon}$ the set of the solids contained in $\Omega_{\varepsilon}$. Then, $S_{\varepsilon}$ is a finite union of solids, i.e. $S_{\varepsilon}=\bigcup_{k^{\prime} \in \mathcal{K}_{\varepsilon}} \bar{Y}_{s_{k^{\prime}}, a_{\varepsilon}}^{\varepsilon}$.

We define $\widetilde{\Omega}_{\varepsilon}=\omega_{\varepsilon} \times(0,1), \Omega=\omega \times(0,1)$, and $Q_{\varepsilon}=\omega \times(0, \varepsilon)$. We observe that $\widetilde{\Omega}_{\varepsilon}=\Omega \backslash \bigcup_{k^{\prime} \in \mathcal{K}_{\varepsilon}} \bar{Y}_{s_{k^{\prime}}, a_{\varepsilon}}$, and we define $T_{\varepsilon}=\bigcup_{k^{\prime} \in \mathcal{K}_{\varepsilon}} \bar{Y}_{s_{k^{\prime}}, a_{\varepsilon}}$ as the set of the solids contained in $\widetilde{\Omega}_{\varepsilon}$.

We remark that along this paper, the points $x \in \mathbb{R}^{3}$ will be decomposed as $x=\left(x^{\prime}, x_{3}\right)$ with $x^{\prime} \in \mathbb{R}^{2}, x_{3} \in \mathbb{R}$. We also use the notation $x^{\prime}$ to denote a generic vector of $\mathbb{R}^{2}$. $\mathbb{Z}^{2}$ by

In order to apply the unfolding method, we will need the following notation. For $k^{\prime} \in \mathbb{Z}^{2}$, we define $\kappa: \mathbb{R}^{2} \rightarrow$

$$
\kappa\left(x^{\prime}\right)=k^{\prime} \Longleftrightarrow x^{\prime} \in Y_{k^{\prime}, 1}^{\prime} .
$$

Remark that $\kappa$ is well defined up to a set of zero measure in $\mathbb{R}^{2}$ (the set $\cup_{k^{\prime} \in \mathbb{Z}^{2}} \partial Y_{k^{\prime}, 1}^{\prime}$ ). Moreover, for every $a_{\varepsilon}>0$, we have

$$
\kappa\left(\frac{x^{\prime}}{a_{\varepsilon}}\right)=k^{\prime} \Longleftrightarrow x^{\prime} \in Y_{k^{\prime}, a_{\varepsilon}}^{\prime} .
$$

The symbol \# means $Y^{\prime}$-periodicity. Thus, for example, the spaces $L_{\#}^{2}(Y), H_{\#}^{1}(Y), H_{0, \#}^{1}(Y)$ are defined by

$$
\begin{gathered}
L_{\#}^{2}(Y)=\left\{\varphi \in L_{\mathrm{loc}}^{2}\left(\mathbb{R}^{2} \times(0,1)\right): \int_{Y}|\varphi|^{2} d y<+\infty, \quad \varphi\left(y^{\prime}+k^{\prime}, y_{3}\right)=\varphi(y), \quad \forall k^{\prime} \in \mathbb{R}^{2}, \text { a.e. } y \in\left(\mathbb{R}^{2} \times(0,1)\right\},\right. \\
H_{\#}^{1}(Y)=\left\{\varphi \in H_{\mathrm{loc}}^{1}\left(\mathbb{R}^{2} \times(0,1)\right): \varphi \in L_{\#}^{2}(Y), D \varphi \in L_{\#}^{2}(Y)^{3}\right\}, \\
H_{0, \#}^{1}(Y)=\left\{\varphi \in H_{\#}^{1}(Y): \varphi=0 \text { on } y_{3}=0,1\right\} .
\end{gathered}
$$

We denote by : the full contraction of two matrices; for $A=\left(a_{i j}\right)_{1 \leq i, j \leq 3}$ and $B=\left(b_{i j}\right)_{1 \leq i, j \leq 3}$, we have $A: B=$ $\sum_{i, j=1}^{3} a_{i j} b_{i j}$

We denote by $O_{\varepsilon}$ a generic real sequence which tends to zero with $\varepsilon$ and can change from line to line. We denote by $C$ a generic constant which can change from line to line.

The problem. In $\Omega_{\varepsilon}$ we consider the stationary flow of an incompressible micropolar fluid which is governed by the following linearized micropolar system formulated in a non-dimensional form (see [17] for more details)

$$
\left\{\begin{aligned}
-\operatorname{div}\left(D u_{\varepsilon}\right)+\nabla p_{\varepsilon}=2 N^{2} \operatorname{rot} w_{\varepsilon}+f_{\varepsilon} & \text { in } \Omega_{\varepsilon}, \\
\operatorname{div} u_{\varepsilon}=0 & \text { in } \Omega_{\varepsilon}, \\
-R_{M} \operatorname{div}\left(D w_{\varepsilon}\right)+4 N^{2} w_{\varepsilon}=2 N^{2} \operatorname{rot} u_{\varepsilon}+g_{\varepsilon} & \text { in } \Omega_{\varepsilon},
\end{aligned}\right.
$$

with homogeneous boundary conditions (it does not alter the generality of the problem under consideration),

$$
u_{\varepsilon}=w_{\varepsilon}=0 \quad \text { on } \quad \partial Q_{\varepsilon} \cup \partial S_{\varepsilon} .
$$

In system (2.5), the velocity $u_{\varepsilon}$, the pressure $p_{\varepsilon}$ and the microrotation $w_{\varepsilon}$ (i.e. the angular velocity field of rotation of particles) are unknown. Observe that equation of the linear momentum (2.5) 1 has the familiar form of the Stokes equation but it is coupled with equation of the angular momentum (2.5) 3 , which esentially describes the motion of the particles inside the microvolume as they undergo microrotational effects represented by the microrotational vector $w_{\varepsilon}$. Dimensionless (non-Newtonian) parameter $N^{2}$ with $0 \leq N \leq 1$ is called coupling number and it characterizes the coupling of the linear and angular momentum equations. When $N$ is identically zero, the equations are decoupled and equation of the linear momentum reduces to the classical Stokes equations which represent Newtonian fluids. The parameter $R_{M}$ is called characteristic length and it characterizes the 
interaction between the micropolar fluid and the microgeometry of the domain. It is small and has to be related to the other small geometrical parameters depending on the type of TPM.

Under assumptions that $f_{\varepsilon}, g_{\varepsilon} \in L^{2}\left(\Omega_{\varepsilon}\right)^{3}$, it is well known that problem (2.5)-(2.6) has a unique weak solution $\left(u_{\varepsilon}, w_{\varepsilon}, p_{\varepsilon}\right) \in H_{0}^{1}\left(\Omega_{\varepsilon}\right)^{3} \times H_{0}^{1}\left(\Omega_{\varepsilon}\right)^{3} \times L_{0}^{2}\left(\Omega_{\varepsilon}\right)$ (see [17]), where the space $L_{0}^{2}\left(\Omega_{\varepsilon}\right)$ is the space of functions of $L^{2}\left(\Omega_{\varepsilon}\right)$ with null integral.

Our aim is to study the asymptotic behavior of $u_{\varepsilon}, w_{\varepsilon}$ and $p_{\varepsilon}$ when $\varepsilon$ and $a_{\varepsilon}$ tend to zero and identify homogenized models coupling the effects of the thickness of the domain and its microgeometry. For this purpose, as usual when we deal with thin domains, we use the dilatation in the variable $x_{3}$ given by

$$
y_{3}=\frac{x_{3}}{\varepsilon},
$$

in order to have the functions defined in the open set with fixed height $\widetilde{\Omega}_{\varepsilon}$.

Namely, we define $\tilde{u}_{\varepsilon}, \tilde{w}_{\varepsilon} \in H_{0}^{1}\left(\widetilde{\Omega}_{\varepsilon}\right)^{3}$ and $\tilde{p}_{\varepsilon} \in L_{0}^{2}\left(\widetilde{\Omega}_{\varepsilon}\right)$ by

$$
\tilde{u}_{\varepsilon}\left(x^{\prime}, y_{3}\right)=u_{\varepsilon}\left(x^{\prime}, \varepsilon y_{3}\right), \quad \tilde{w}_{\varepsilon}\left(x^{\prime}, y_{3}\right)=w_{\varepsilon}\left(x^{\prime}, \varepsilon y_{3}\right), \quad \tilde{p}_{\varepsilon}\left(x^{\prime}, y_{3}\right)=p_{\varepsilon}\left(x^{\prime}, \varepsilon y_{3}\right), \quad \text { a.e. }\left(x^{\prime}, y_{3}\right) \in \widetilde{\Omega}_{\varepsilon} \text {. }
$$

Let us introduce some notation which will be useful in the following. For a vectorial function $v=\left(v^{\prime}, v_{3}\right)$ and a scalar function $w$, we introduce the operators $D_{\varepsilon}, \nabla_{\varepsilon}$ and $\operatorname{rot}_{\varepsilon}$ by

$$
\begin{gathered}
\left(D_{\varepsilon} v\right)_{i j}=\partial_{x_{j}} v_{i} \text { for } i=1,2,3, j=1,2, \quad\left(D_{\varepsilon} v\right)_{i, 3}=\frac{1}{\varepsilon} \partial_{y_{3}} v_{i} \text { for } i=1,2,3, \\
\nabla_{\varepsilon} w=\left(\nabla_{x^{\prime}} w, \frac{1}{\varepsilon} \partial_{y_{3}} w\right)^{t}, \quad \operatorname{div}_{\varepsilon} v=\operatorname{div}_{x^{\prime}} v^{\prime}+\frac{1}{\varepsilon} \partial_{y_{3}} v_{3}, \quad \operatorname{rot}_{\varepsilon} v=\left(\operatorname{rot}_{x^{\prime}} v_{3}+\frac{1}{\varepsilon} \operatorname{rot}_{y_{3}} v^{\prime}, \operatorname{Rot}_{x^{\prime}} v^{\prime}\right)^{t},
\end{gathered}
$$

where, denoting $\left(v^{\prime}\right)^{\perp}=\left(-v_{2}, v_{1}\right)^{t}$, we define

$$
\operatorname{rot}_{x^{\prime}} v_{3}=\left(\partial_{x_{2}} v_{3},-\partial_{x_{1}} v_{3}\right)^{t}, \quad \operatorname{rot}_{y_{3}} v^{\prime}=\left(\partial_{y_{3}} v^{\prime}\right)^{\perp}, \quad \operatorname{Rot}_{x^{\prime}} v^{\prime}=\partial_{x_{1}} v_{2}-\partial_{x_{2}} v_{1} .
$$

Using the transformation (2.7), the rescaled system (2.5)-(2.6) can be rewritten as

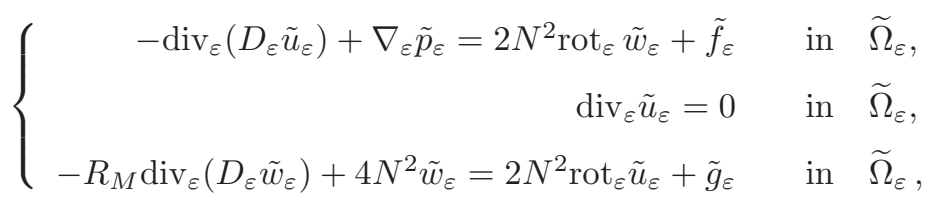

with homogeneous boundary conditions

$$
\tilde{u}_{\varepsilon}=\tilde{w}_{\varepsilon}=0 \quad \text { on } \quad \partial \Omega \cup \partial T_{\varepsilon},
$$

where $\tilde{f}_{\varepsilon}$ and $\tilde{g}_{\varepsilon}$ are defined similarly as in (2.8).

Our goal then is to describe the asymptotic behavior of this new sequences $\tilde{u}_{\varepsilon}, \tilde{w}_{\varepsilon}$ and $\tilde{p}_{\varepsilon}$ when $\varepsilon$ and $a_{\varepsilon}$ tend to zero. For this, it will be useful to use the the equivalent weak variational formulation of system (2.5)-(2.6) and the rescaled system (2.10)-(2.11).

Weak variational formulations. For problem (2.5)-(2.6), the weak variational formulation is to find $u_{\varepsilon}, w_{\varepsilon} \in$ $H_{0}^{1}\left(\Omega_{\varepsilon}\right)^{3}$ and $p_{\varepsilon} \in L_{0}^{2}\left(\Omega_{\varepsilon}\right)$ such that

$$
\left\{\begin{array}{l}
\int_{\Omega_{\varepsilon}} D u_{\varepsilon}: D \varphi d x-\int_{\Omega_{\varepsilon}} p_{\varepsilon} \operatorname{div} \varphi d x=2 N^{2} \int_{\Omega_{\varepsilon}} \operatorname{rot} w_{\varepsilon} \cdot \varphi d x+\int_{\Omega_{\varepsilon}} f_{\varepsilon} \cdot \varphi d x, \\
R_{M} \int_{\Omega_{\varepsilon}} D w_{\varepsilon}: D \psi d x+4 N^{2} \int_{\Omega_{\varepsilon}} w_{\varepsilon} \cdot \psi d x=2 N^{2} \int_{\Omega_{\varepsilon}} \operatorname{rot} u_{\varepsilon} \cdot \psi d x+\int_{\Omega_{\varepsilon}} g_{\varepsilon} \cdot \psi d x,
\end{array}\right.
$$

for every $\varphi, \psi \in H_{0}^{1}\left(\Omega_{\varepsilon}\right)^{3}$, and the equivalent weak variational formulation for the rescaled system (2.10)-(2.11) is to find $\tilde{u}_{\varepsilon}, \tilde{w}_{\varepsilon} \in H_{0}^{1}\left(\widetilde{\Omega}_{\varepsilon}\right)^{3}$ and $\tilde{p}_{\varepsilon} \in L_{0}^{2}\left(\widetilde{\Omega}_{\varepsilon}\right)$ such that 


$$
\left\{\begin{array}{l}
\int_{\widetilde{\Omega}_{\varepsilon}} D_{\varepsilon} \tilde{u}_{\varepsilon}: D_{\varepsilon} \varphi d x^{\prime} d y_{3}-\int_{\widetilde{\Omega}_{\varepsilon}} \tilde{p}_{\varepsilon} \operatorname{div}_{\varepsilon} \varphi d x^{\prime} d y_{3}=2 N^{2} \int_{\widetilde{\Omega}_{\varepsilon}} \operatorname{rot}_{\varepsilon} \tilde{w}_{\varepsilon} \cdot \varphi d x^{\prime} d y_{3}+\int_{\widetilde{\Omega}_{\varepsilon}} \tilde{f}_{\varepsilon} \cdot \varphi d x^{\prime} d y_{3}, \\
R_{M} \int_{\widetilde{\Omega}_{\varepsilon}} D_{\varepsilon} \tilde{w}_{\varepsilon}: D_{\varepsilon} \psi d x^{\prime} d y_{3}+4 N^{2} \int_{\widetilde{\Omega}_{\varepsilon}} \tilde{w}_{\varepsilon} \cdot \psi d x^{\prime} d y_{3}=2 N^{2} \int_{\widetilde{\Omega}_{\varepsilon}} \operatorname{rot}_{\varepsilon} \tilde{u}_{\varepsilon} \cdot \psi d x^{\prime} d y_{3}+\int_{\widetilde{\Omega}_{\varepsilon}} \tilde{g}_{\varepsilon} \cdot \psi d x^{\prime} d y_{3},
\end{array}\right.
$$

for every $\varphi, \psi \in H_{0}^{1}\left(\widetilde{\Omega}_{\varepsilon}\right)^{3}$.

\section{$3 \quad$ A priori estimates}

In the sequel we make the following assumptions concerning $f_{\varepsilon}, g_{\varepsilon}, R_{M}$ and $N$ :

i) in the cases PTPM and HTPM, we assume

$$
\begin{gathered}
f_{\varepsilon}(x)=\left(f^{\prime}\left(x^{\prime}\right), 0\right), \quad g_{\varepsilon}(x)=\left(a_{\varepsilon} g^{\prime}\left(x^{\prime}\right), 0\right), \quad \text { a.e. } x \in \Omega_{\varepsilon}, \quad \text { where } f^{\prime}, g^{\prime} \in L^{2}(\omega)^{2}, \\
N^{2}=\mathcal{O}(1), \quad R_{M}=a_{\varepsilon}^{2} R_{c} \quad \text { with } R_{c}=\mathcal{O}(1),
\end{gathered}
$$

ii) in the case VTPM, we assume

$$
\begin{gathered}
f_{\varepsilon}(x)=\left(f^{\prime}\left(x^{\prime}\right), 0\right), \quad g_{\varepsilon}(x)=\left(\varepsilon g^{\prime}\left(x^{\prime}\right), 0\right), \quad \text { a.e. } x \in \Omega_{\varepsilon}, \quad \text { where } f^{\prime}, g^{\prime} \in L^{2}(\omega)^{2}, \\
N^{2}=\mathcal{O}(1), \quad R_{M}=\varepsilon^{2} R_{c} \quad \text { with } R_{c}=\mathcal{O}(1) .
\end{gathered}
$$

Remark 3.1. We point out that in PTPM and HTPM the parameter $R_{M}$ is compared with the size of the obstacles while in the case VTPM with the film thickness, which are the most challenging ones and they answer to the question addressed in the paper, all preserve in the limit a strong coupling between velocity and microrotation. This choice is justified by many studies, for example in the selected applications chapter in [17] (see also [7, 8]).

We also observe that due to the thickness of the domain, it is usual to assume that the vertical components of $f$ and $g$ can be neglected and, moreover they can be considered independent of the vertical variable. The parameters for $g_{\varepsilon}$ are chosen to obtain appropriate estimates in each case.

First, we recall the Poincaré inequality in a thin porous medium domain $\Omega_{\varepsilon}$ (see [4).

Lemma 3.2. There exists a constant $C$ independent of $\varepsilon$, such that,

i) in the cases PTPM and HTPM, then

$$
\|v\|_{L^{2}\left(\Omega_{\varepsilon}\right)^{3}} \leq C a_{\varepsilon}\|D v\|_{L^{2}\left(\Omega_{\varepsilon}\right)^{3 \times 3}}, \quad \forall v \in H_{0}^{1}\left(\Omega_{\varepsilon}\right)^{3},
$$

ii) in the case VTPM, then

$$
\|v\|_{L^{2}\left(\Omega_{\varepsilon}\right)^{3}} \leq C \varepsilon\|D v\|_{L^{2}\left(\Omega_{\varepsilon}\right)^{3 \times 3}}, \quad \forall v \in H_{0}^{1}\left(\Omega_{\varepsilon}\right)^{3} .
$$

Next, we give the following results relating the derivative and the rotational (see [1]).

Lemma 3.3. The following inequality holds

$$
\|\operatorname{rot} v\|_{L^{2}\left(\Omega_{\varepsilon}\right)^{3}} \leq\|D v\|_{L^{2}\left(\Omega_{\varepsilon}\right)^{3 \times 3}}, \quad \forall v \in H_{0}^{1}\left(\Omega_{\varepsilon}\right)^{3} .
$$

Moreover, if $\operatorname{div} v=0$ in $\Omega_{\varepsilon}$, then it holds

$$
\|\operatorname{rot} v\|_{L^{2}\left(\Omega_{\varepsilon}\right)^{3}}=\|D v\|_{L^{2}\left(\Omega_{\varepsilon}\right)^{3 \times 3}} .
$$


We start by obtaining some a priori estimates for $\tilde{u}_{\varepsilon}$ and $\tilde{w}_{\varepsilon}$.

Lemma 3.4. There exists a constant $C$ independent of $\varepsilon$, such that the rescaled solution $\left(\tilde{u}_{\varepsilon}, \tilde{w}_{\varepsilon}\right)$ of the problem (2.10)-(2.11) satisfies

i) in the cases PTPM and HTPM,

$$
\begin{array}{ll}
\left\|\tilde{u}_{\varepsilon}\right\|_{L^{2}\left(\widetilde{\Omega}_{\varepsilon}\right)^{3}} \leq C a_{\varepsilon}^{2}, \quad\left\|D_{\varepsilon} \tilde{u}_{\varepsilon}\right\|_{L^{2}\left(\widetilde{\Omega}_{\varepsilon}\right)^{3 \times 3}} \leq C a_{\varepsilon}, \\
\left\|\tilde{w}_{\varepsilon}\right\|_{L^{2}\left(\widetilde{\Omega}_{\varepsilon}\right)^{3}} \leq C a_{\varepsilon}, \quad\left\|D_{\varepsilon} \tilde{w}_{\varepsilon}\right\|_{L^{2}\left(\widetilde{\Omega}_{\varepsilon}\right)^{3 \times 3}} \leq C .
\end{array}
$$

ii) in the case VTPM,

$$
\begin{array}{cl}
\left\|\tilde{u}_{\varepsilon}\right\|_{L^{2}\left(\widetilde{\Omega}_{\varepsilon}\right)^{3}} \leq C \varepsilon^{2}, & \left\|D_{\varepsilon} \tilde{u}_{\varepsilon}\right\|_{L^{2}\left(\widetilde{\Omega}_{\varepsilon}\right)^{3 \times 3}} \leq C \varepsilon, \\
\left\|\tilde{w}_{\varepsilon}\right\|_{L^{2}\left(\widetilde{\Omega}_{\varepsilon}\right)^{3}} \leq C \varepsilon, \quad\left\|D_{\varepsilon} \tilde{w}_{\varepsilon}\right\|_{L^{2}\left(\widetilde{\Omega}_{\varepsilon}\right)^{3 \times 3}} \leq C .
\end{array}
$$

Proof. We analyze the different cases.

i) Cases PTPM and HTPM. We first obtain the estimates for the velocity. Taking $\varphi=u_{\varepsilon}$ as test function in the first equation of (2.12), taking into account $\int_{\Omega_{\varepsilon}} \operatorname{rot} w_{\varepsilon} \cdot u_{\varepsilon} d x=\int_{\Omega_{\varepsilon}} \operatorname{rot} u_{\varepsilon} \cdot w_{\varepsilon} d x$, applying CauchySchwarz's inequality and from Lemma 3.2 and (3.21), we have

$$
\begin{aligned}
\left\|D u_{\varepsilon}\right\|_{L^{2}\left(\Omega_{\varepsilon}\right)^{3 \times 3}}^{2} & =2 N^{2} \int_{\Omega_{\varepsilon}} \operatorname{rot} w_{\varepsilon} \cdot u_{\varepsilon} d x+\int_{\Omega_{\varepsilon}} f_{\varepsilon} \cdot u_{\varepsilon} d x \\
& =2 N^{2} \int_{\Omega_{\varepsilon}} w_{\varepsilon} \cdot \operatorname{rot} u_{\varepsilon} d x+\int_{\Omega_{\varepsilon}} f^{\prime}\left(x^{\prime}\right) \cdot u_{\varepsilon}^{\prime} d x \\
& \leq 2 N^{2}\left\|w_{\varepsilon}\right\|_{L^{2}\left(\Omega_{\varepsilon}\right)^{3}}\left\|D u_{\varepsilon}\right\|_{L^{2}\left(\Omega_{\varepsilon}\right)^{3 \times 3}}+\varepsilon^{\frac{1}{2}} a_{\varepsilon} C\left\|f^{\prime}\right\|_{L^{2}(\omega)^{2}}\left\|D u_{\varepsilon}\right\|_{L^{2}\left(\Omega_{\varepsilon}\right)^{3 \times 3}},
\end{aligned}
$$

which implies

$$
\varepsilon^{-\frac{1}{2}} a_{\varepsilon}^{-1}\left\|D u_{\varepsilon}\right\|_{L^{2}\left(\Omega_{\varepsilon}\right)^{3 \times 3}} \leq \varepsilon^{-\frac{1}{2}} a_{\varepsilon}^{-1} 2 N^{2}\left\|w_{\varepsilon}\right\|_{L^{2}\left(\Omega_{\varepsilon}\right)^{3}}+C\left\|f^{\prime}\right\|_{L^{2}(\omega)^{2}} .
$$

Taking now $\psi=w_{\varepsilon}$ as test function in the second equation of (2.12), applying Cauchy-Schwarz's inequality and taking into account (3.14) and (3.15), we have

$$
\begin{aligned}
& a_{\varepsilon}^{2} R_{c}\left\|D w_{\varepsilon}\right\|_{L^{2}\left(\Omega_{\varepsilon}\right)^{3 \times 3}}^{2}+4 N^{2}\left\|w_{\varepsilon}\right\|_{L^{2}\left(\Omega_{\varepsilon}\right)^{3}}^{2} \\
& \quad=2 N^{2} \int_{\Omega_{\varepsilon}} \operatorname{rot} u_{\varepsilon} \cdot w_{\varepsilon} d x+a_{\varepsilon} \int_{\Omega_{\varepsilon}} g^{\prime}\left(x^{\prime}\right) \cdot w_{\varepsilon}^{\prime} d x \\
& \quad \leq 2 N^{2}\left\|w_{\varepsilon}\right\|_{L^{2}\left(\Omega_{\varepsilon}\right)^{3}}\left\|D u_{\varepsilon}\right\|_{L^{2}\left(\Omega_{\varepsilon}\right)^{3 \times 3}}+\varepsilon^{\frac{1}{2}} a_{\varepsilon}\left\|g^{\prime}\right\|_{L^{2}(\omega)^{2}}\left\|w_{\varepsilon}\right\|_{L^{2}\left(\Omega_{\varepsilon}\right)^{3}},
\end{aligned}
$$

which implies

$$
\varepsilon^{-\frac{1}{2}} a_{\varepsilon}^{-1} 2 N^{2}\left\|w_{\varepsilon}\right\|_{L^{2}\left(\Omega_{\varepsilon}\right)^{3}} \leq \varepsilon^{-\frac{1}{2}} a_{\varepsilon}^{-1} N^{2}\left\|D u_{\varepsilon}\right\|_{L^{2}\left(\Omega_{\varepsilon}\right)^{3 \times 3}}+\frac{1}{2}\left\|g^{\prime}\right\|_{L^{2}(\omega)^{2}} .
$$

Then, from (3.27) and (3.29), we conclude that

$$
\varepsilon^{-\frac{1}{2}} a_{\varepsilon}^{-1}\left\|D u_{\varepsilon}\right\|_{L^{2}\left(\Omega_{\varepsilon}\right)^{3 \times 3}} \leq \frac{C}{1-N^{2}}\left\|f^{\prime}\right\|_{L^{2}(\omega)^{2}}+\frac{1}{2\left(1-N^{2}\right)}\left\|g^{\prime}\right\|_{L^{2}(\omega)^{2}},
$$

which gives

$$
\left\|D u_{\varepsilon}\right\|_{L^{2}\left(\Omega_{\varepsilon}\right)^{3 \times 3}} \leq C a_{\varepsilon} \varepsilon^{\frac{1}{2}} .
$$

This together with Lemma 3.2 gives

$$
\left\|u_{\varepsilon}\right\|_{L^{2}\left(\Omega_{\varepsilon}\right)^{3}} \leq C a_{\varepsilon}^{2} \varepsilon^{\frac{1}{2}}
$$


and by means of the dilatation (2.7) we get (3.22).

Finally, we obtain the estimates for the microrotation. We use $\int_{\Omega_{\varepsilon}} \operatorname{rot} u_{\varepsilon} \cdot w_{\varepsilon} d x=\int_{\Omega_{\varepsilon}} \operatorname{rot} w_{\varepsilon} \cdot u_{\varepsilon} d x$ in (3.28), Lemma 3.2 and (3.20), and proceeding as above we obtain

$$
\begin{aligned}
& a_{\varepsilon}^{2} R_{c}\left\|D w_{\varepsilon}\right\|_{L^{2}\left(\Omega_{\varepsilon}\right)^{3 \times 3}}^{2}+4 N^{2}\left\|w_{\varepsilon}\right\|_{L^{2}\left(\Omega_{\varepsilon}\right)^{3}}^{2} \\
& \quad \leq 2 N^{2}\left\|u_{\varepsilon}\right\|_{L^{2}\left(\Omega_{\varepsilon}\right)^{3}}\left\|D w_{\varepsilon}\right\|_{L^{2}\left(\Omega_{\varepsilon}\right)^{3 \times 3}}+\varepsilon^{\frac{1}{2}} a_{\varepsilon}^{2} C\left\|g^{\prime}\right\|_{L^{2}(\omega)^{2}}\left\|D w_{\varepsilon}\right\|_{L^{2}\left(\Omega_{\varepsilon}\right)^{3 \times 3}},
\end{aligned}
$$

which, by using the estimate of $u_{\varepsilon}$ given in (3.30), provides

$$
a_{\varepsilon}^{2} R_{c}\left\|D w_{\varepsilon}\right\|_{L^{2}\left(\Omega_{\varepsilon}\right)^{3 \times 3}} \leq C\left(2 N^{2} \varepsilon^{\frac{1}{2}} a_{\varepsilon}^{2}+\varepsilon^{\frac{1}{2}} a_{\varepsilon}^{2}\left\|g^{\prime}\right\|_{L^{2}(\omega)^{2}}\right) .
$$

This implies

$$
\left\|w_{\varepsilon}\right\|_{L^{2}\left(\Omega_{\varepsilon}\right)^{3}} \leq C a_{\varepsilon} \varepsilon^{\frac{1}{2}}, \quad\left\|D w_{\varepsilon}\right\|_{L^{2}\left(\Omega_{\varepsilon}\right)^{3 \times 3}} \leq C \varepsilon^{\frac{1}{2}},
$$

and by means of the dilatation, we get (3.23).

ii) Case VTPM. For the velocity, proceeding as above by taking into account (3.16) and (3.17), we conclude

$$
\varepsilon^{-\frac{3}{2}}\left\|D u_{\varepsilon}\right\|_{L^{2}\left(\Omega_{\varepsilon}\right)^{3 \times 3}} \leq \varepsilon^{-\frac{3}{2}} 2 N^{2}\left\|w_{\varepsilon}\right\|_{L^{2}\left(\Omega_{\varepsilon}\right)^{3}}+C\left\|f^{\prime}\right\|_{L^{2}(\omega)^{2}}
$$

and

$$
\varepsilon^{-\frac{3}{2}} 2 N^{2}\left\|w_{\varepsilon}\right\|_{L^{2}\left(\Omega_{\varepsilon}\right)^{3}} \leq \varepsilon^{-\frac{3}{2}} N^{2}\left\|D u_{\varepsilon}\right\|_{L^{2}\left(\Omega_{\varepsilon}\right)^{3 \times 3}}+\frac{1}{2}\left\|g^{\prime}\right\|_{L^{2}(\omega)^{2}} .
$$

Then, we deduce estimate

$$
\left\|u_{\varepsilon}\right\|_{L^{2}\left(\Omega_{\varepsilon}\right)^{3}} \leq C \varepsilon^{\frac{5}{2}}, \quad\left\|D u_{\varepsilon}\right\|_{L^{2}\left(\Omega_{\varepsilon}\right)^{3 \times 3}} \leq C \varepsilon^{\frac{3}{2}}
$$

and by means of the dilatation, we get (3.24).

For the microrotation, similarly as the previous case, we get that

$$
\varepsilon^{2} R_{c}\left\|D w_{\varepsilon}\right\|_{L^{2}\left(\Omega_{\varepsilon}\right)^{3 \times 3}} \leq C\left(2 N^{2} \varepsilon^{\frac{5}{2}}+\varepsilon^{\frac{5}{2}}\left\|g^{\prime}\right\|_{L^{2}(\omega)^{2}}\right),
$$

which implies

$$
\left\|w_{\varepsilon}\right\|_{L^{2}\left(\Omega_{\varepsilon}\right)^{3}} \leq C \varepsilon^{\frac{3}{2}}, \quad\left\|D w_{\varepsilon}\right\|_{L^{2}\left(\Omega_{\varepsilon}\right)^{3 \times 3}} \leq C \varepsilon^{\frac{1}{2}},
$$

and by means of the dilatation we get (3.25).

\subsection{The extension of $\left(\tilde{u}_{\varepsilon}, \tilde{w}_{\varepsilon}, \tilde{p}_{\varepsilon}\right)$ to the whole domain $\Omega$}

We extend the velocity $\tilde{u}_{\varepsilon}$ and the microrotation $\tilde{w}_{\varepsilon}$ by zero to the $\Omega \backslash \widetilde{\Omega}_{\varepsilon}$, and denote the extension by the same symbol. Obviously, estimates (3.22)-(3.25) remain valid and the extension of $\tilde{u}_{\varepsilon}$ is divergence free too.

In order to extend the pressure to the whole domain $\Omega$, we use the mapping $R^{\varepsilon}$, defined in Lemma 4.5 in 4 ] as $R_{2}^{\varepsilon}$, which allows us to extend the pressure $p_{\varepsilon}$ from $\Omega_{\varepsilon}$ to $Q_{\varepsilon}$ by introducing $F_{\varepsilon}$ in $H^{-1}\left(Q_{\varepsilon}\right)^{3}$ in the following way (brackets are for the duality products between $H^{-1}$ and $H_{0}^{1}$ ):

$$
\left\langle F_{\varepsilon}, \varphi\right\rangle_{Q_{\varepsilon}}=\left\langle\nabla p_{\varepsilon}, R^{\varepsilon} \varphi\right\rangle_{\Omega_{\varepsilon}}, \quad \text { for any } \varphi \in H_{0}^{1}\left(Q_{\varepsilon}\right)^{3} .
$$

Using Lemma 3.4 for fixed $\varepsilon$ and $a_{\varepsilon}$, we see that it i a bounded functional on $H_{0}^{1}\left(Q_{\varepsilon}\right.$ ) (see the proof of Lemma 3.5 below), and in fact $F_{\varepsilon} \in H^{-1}\left(Q_{\varepsilon}\right)^{3}$. Moreover, $\operatorname{div} \varphi=0$ implies $\left\langle F_{\varepsilon}, \varphi\right\rangle_{Q_{\varepsilon}}=0$, and the DeRham theorem gives the existence of $P_{\varepsilon}$ in $L_{0}^{2}\left(Q_{\varepsilon}\right)$ with $F_{\varepsilon}=\nabla P_{\varepsilon}$. 
We calcule the right hand side of (3.32) by using the first equation of (2.12) and we have

$$
\left\langle F_{\varepsilon}, \varphi\right\rangle_{Q_{\varepsilon}}=-\int_{\Omega_{\varepsilon}} D u_{\varepsilon}: D R^{\varepsilon} \varphi d x+2 N^{2} \int_{\Omega_{\varepsilon}} \operatorname{rot} w_{\varepsilon} \cdot R^{\varepsilon} \varphi d x+\int_{\Omega_{\varepsilon}} f^{\prime}\left(x^{\prime}\right) \cdot\left(R^{\varepsilon} \varphi\right)^{\prime} d x .
$$

We get for any $\tilde{\varphi} \in H_{0}^{1}(\Omega)^{3}$ where $\tilde{\varphi}\left(x^{\prime}, y_{3}\right)=\varphi\left(x^{\prime}, \varepsilon y_{3}\right)$, using the change of variables (2.7), that

$$
\left\langle\nabla_{\varepsilon} \tilde{P}_{\varepsilon}, \tilde{\varphi}\right\rangle_{\Omega}=-\int_{\Omega} \tilde{P}_{\varepsilon} \operatorname{div}_{\varepsilon} \tilde{\varphi} d x^{\prime} d y_{3}=-\varepsilon^{-1} \int_{Q_{\varepsilon}} P_{\varepsilon} \operatorname{div} \varphi d x=\varepsilon^{-1}\left\langle\nabla P_{\varepsilon}, \varphi\right\rangle_{Q_{\varepsilon}} .
$$

Then, using the identification (3.33) of $F_{\varepsilon}$, we have

$$
\left\langle\nabla_{\varepsilon} \tilde{P}_{\varepsilon}, \tilde{\varphi}\right\rangle_{\Omega}=\varepsilon^{-1}\left(-\int_{\Omega_{\varepsilon}} D u_{\varepsilon}: D R^{\varepsilon} \varphi d x+2 N^{2} \int_{\Omega_{\varepsilon}} \operatorname{rot} w_{\varepsilon} \cdot R^{\varepsilon} \varphi d x+\int_{\Omega_{\varepsilon}} f^{\prime}\left(x^{\prime}\right) \cdot\left(R^{\varepsilon} \varphi\right)^{\prime} d x\right),
$$

and applying the change of variables (2.7), we obtain

$$
\left\langle\nabla_{\varepsilon} \tilde{P}_{\varepsilon}, \tilde{\varphi}\right\rangle_{\Omega}=-\int_{\widetilde{\Omega}_{\varepsilon}} D_{\varepsilon} \tilde{u}_{\varepsilon}: D_{\varepsilon} \tilde{R}^{\varepsilon} \tilde{\varphi} d x^{\prime} d y_{3}+2 N^{2} \int_{\widetilde{\Omega}_{\varepsilon}} \operatorname{rot}_{\varepsilon} \tilde{w}_{\varepsilon} \cdot \tilde{R}^{\varepsilon} \tilde{\varphi} d x^{\prime} d y_{3}+\int_{\widetilde{\Omega}_{\varepsilon}} f\left(x^{\prime}\right) \cdot\left(\tilde{R}^{\varepsilon} \tilde{\varphi}\right)^{\prime} d x^{\prime} d y_{3},
$$

where $\tilde{R}^{\varepsilon} \tilde{\varphi}=R^{\varepsilon} \varphi$ for any $\tilde{\varphi} \in H_{0}^{1}(\Omega)^{3}$.

Now, we estimate the right-hand side of (3.34).

Lemma 3.5. There exists a constant $C>0$ independent of $\varepsilon$, such that the extension $\tilde{P}_{\varepsilon} \in L_{0}^{2}(\Omega)$ of the pressure $\tilde{p}_{\varepsilon}$ satisfies

$$
\left\|\tilde{P}_{\varepsilon}\right\|_{L_{0}^{2}(\Omega)} \leq C
$$

Proof. From the proof of Lemma 4.6-(i) in [4, we have that $\tilde{R}^{\varepsilon} \tilde{\varphi}$ satisfies the following estimates

$$
\left\{\begin{array}{l}
\left\|\tilde{R}^{\varepsilon}(\tilde{\varphi})\right\|_{L^{2}\left(\widetilde{\Omega}_{\varepsilon}\right)^{3}}^{2} \leq C\left(\frac{1}{a_{\varepsilon}^{2}}\|\tilde{\varphi}\|_{L^{2}(\Omega)^{3}}^{2}+\left\|D_{x^{\prime}} \tilde{\varphi}\right\|_{L^{2}(\Omega)^{3 \times 2}}^{2}+\frac{1}{a_{\varepsilon}^{2}}\left\|\partial_{y_{3}} \tilde{\varphi}\right\|_{L^{2}(\Omega)^{3}}^{2}\right) \leq C\|\tilde{\varphi}\|_{H_{0}^{1}(\Omega)^{3}}, \\
\left\|D_{x^{\prime}} \tilde{R}^{\varepsilon} \tilde{\varphi}\right\|_{L^{2}\left(\widetilde{\Omega}_{\varepsilon}\right)^{3 \times 2}}^{2} \leq C\left(\frac{1}{a_{\varepsilon}^{2}}\|\tilde{\varphi}\|_{L^{2}(\Omega)^{3}}^{2}+\left\|D_{x^{\prime}} \tilde{\varphi}\right\|_{L^{2}(\Omega)^{3 \times 2}}^{2}+\frac{1}{a_{\varepsilon}^{2}}\left\|\partial_{y_{3}} \tilde{\varphi}\right\|_{L^{2}(\Omega)^{3}}^{2}\right), \\
\left\|\partial_{y_{3}} \tilde{R}^{\varepsilon} \tilde{\varphi}\right\|_{L^{2}\left(\widetilde{\Omega}_{\varepsilon}\right)^{3}}^{2} \leq C\left(\|\tilde{\varphi}\|_{L^{2}(\Omega)^{3}}^{2}+a_{\varepsilon}^{2}\left\|D_{x^{\prime}} \tilde{\varphi}\right\|_{L^{2}(\Omega)^{2 \times 3}}^{2}+\left\|\partial_{y_{3}} \tilde{\varphi}\right\|_{L^{2}(\Omega)^{3}}^{2}\right) .
\end{array}\right.
$$

This implies that

$$
\left\|\tilde{R}^{\varepsilon}(\tilde{\varphi})\right\|_{L^{2}\left(\widetilde{\Omega}_{\varepsilon}\right)^{3}} \leq C\left(\|\tilde{\varphi}\|_{L^{2}(\Omega)^{3}}^{2}+a_{\varepsilon}\left\|D_{x^{\prime}} \tilde{\varphi}\right\|_{L^{2}(\Omega)^{3 \times 2}}+\left\|\partial_{y_{3}} \tilde{\varphi}\right\|_{L^{2}(\Omega)^{3}}^{2}\right) \leq C\|\tilde{\varphi}\|_{H_{0}^{1}(\Omega)^{3}} .
$$

Moreover, in the case PTPM and HTPM,

$$
\left\|D_{\varepsilon} \tilde{R}^{\varepsilon} \tilde{\varphi}\right\|_{L^{2}\left(\widetilde{\Omega}_{\varepsilon}\right)^{3 \times 3}} \leq C\left(\frac{1}{a_{\varepsilon}}\|\tilde{\varphi}\|_{L^{2}(\Omega)^{3}}+\left\|D_{x^{\prime}} \tilde{\varphi}\right\|_{L^{2}(\Omega)^{3 \times 2}}+\frac{1}{a_{\varepsilon}}\left\|\partial_{y_{3}} \tilde{\varphi}\right\|_{L^{2}(\Omega)^{3}}\right) \leq \frac{C}{a_{\varepsilon}}\|\tilde{\varphi}\|_{H_{0}^{1}(\Omega)^{3}},
$$

and in the case VTPM,

$$
\left\|D_{\varepsilon} \tilde{R}^{\varepsilon} \tilde{\varphi}\right\|_{L^{2}\left(\widetilde{\Omega}_{\varepsilon}\right)^{3 \times 3}} \leq C\left(\frac{1}{\varepsilon}\|\tilde{\varphi}\|_{L^{2}(\Omega)^{3}}+\frac{a_{\varepsilon}}{\varepsilon}\left\|D_{x^{\prime}} \tilde{\varphi}\right\|_{L^{2}(\Omega)^{3 \times 3}}+\frac{1}{\varepsilon}\left\|\partial_{y_{3}} \tilde{\varphi}\right\|_{L^{2}(\Omega)^{3}}\right) \leq \frac{C}{\varepsilon}\|\tilde{\varphi}\|_{H_{0}^{1}(\Omega)^{3}}
$$

Thus, in the cases PTMP and HTPM, by using estimates for $D_{\varepsilon} \tilde{u}_{\varepsilon}$ in (3.22), for $D_{\varepsilon} w_{\varepsilon}$ in (3.23) and $f^{\prime} \in L^{2}(\omega)^{2}$, we respectively obtain

$$
\begin{aligned}
& \left|\int_{\widetilde{\Omega}_{\varepsilon}} D_{\varepsilon} \tilde{u}_{\varepsilon}: D_{\varepsilon} \tilde{R}^{\varepsilon} \tilde{\varphi} d x^{\prime} d y_{3}\right| \leq C a_{\varepsilon}\left\|D_{\varepsilon} \tilde{R}^{\varepsilon} \tilde{\varphi}\right\|_{L^{2}\left(\widetilde{\Omega}_{\varepsilon}\right)^{3 \times 3}} \leq C\|\tilde{\varphi}\|_{H_{0}^{1}(\Omega)^{3}}, \\
& \left|\int_{\widetilde{\Omega}_{\varepsilon}} \operatorname{rot}_{\varepsilon} w_{\varepsilon} \cdot \tilde{R}^{\varepsilon} \tilde{\varphi} d x^{\prime} d y_{3}\right| \leq\left\|D_{\varepsilon} \tilde{w}_{\varepsilon}\right\|_{L^{2}\left(\widetilde{\Omega}_{\varepsilon}\right)^{3 \times 3}}\left\|\tilde{R}^{\varepsilon} \tilde{\varphi}\right\|_{L^{2}\left(\widetilde{\Omega}_{\varepsilon}\right)^{3}} \leq C a_{\varepsilon}^{2} \varepsilon^{-2}\left\|\tilde{R}^{\varepsilon} \tilde{\varphi}\right\|_{L^{2}\left(\widetilde{\Omega}_{\varepsilon}\right)^{3}} \leq C\|\tilde{\varphi}\|_{H_{0}^{1}(\Omega)^{3}}, \\
& \left|\int_{\widetilde{\Omega}_{\varepsilon}} f^{\prime} \cdot \tilde{R}^{\varepsilon} \tilde{\varphi} d x^{\prime} d y_{3}\right| \leq C\left\|\tilde{R}^{\varepsilon} \tilde{\varphi}\right\|_{L^{2}\left(\widetilde{\Omega}_{\varepsilon}\right)^{3}} \leq C\|\tilde{\varphi}\|_{H_{0}^{1}(\Omega)^{3}},
\end{aligned}
$$


which together with (3.34) gives $\left\|\nabla_{\varepsilon} \tilde{P}_{\varepsilon}\right\|_{L^{2}(\Omega)^{3}} \leq C$. By using the Nečas inequality there exists a representative $\tilde{P}_{\varepsilon} \in L_{0}^{2}(\Omega)$ such that

$$
\left\|\tilde{P}_{\varepsilon}\right\|_{L^{2}(\Omega)} \leq C\left\|\nabla \tilde{P}_{\varepsilon}\right\|_{H^{-1}(\Omega)^{3}} \leq C\left\|\nabla_{\varepsilon} \tilde{P}_{\varepsilon}\right\|_{H^{-1}(\Omega)^{3}},
$$

which implies (3.35).

Finally, proceeding similarly in the case VTPM, by using now estimates for $D_{\varepsilon} \tilde{u}_{\varepsilon}$ in (3.24) and for $D_{\varepsilon} w_{\varepsilon}$ in (3.25), we also obtain (3.35).

\subsection{Adaptation of the unfolding method}

The change of variables (2.7) does not provide the information we need about the behavior of $\tilde{u}_{\varepsilon}$ and $\tilde{w}_{\varepsilon}$ in the microstructure associated to $\widetilde{\Omega}_{\varepsilon}$. To solve this difficulty, we use an adaptation of the unfolding method (see [6, 9, 10] for more details) introduced in [4].

Let us recall that this adaptation of the unfolding method divides the domain $\widetilde{\Omega}_{\varepsilon}$ in cubes of lateral length $a_{\varepsilon}$ and vertical length 1 . Thus, given $\left(\tilde{u}_{\varepsilon}, \tilde{w}_{\varepsilon}, \tilde{P}_{\varepsilon}\right) \in H_{0}^{1}(\Omega)^{3} \times H_{0}^{1}(\Omega)^{3} \times L_{0}^{2}(\Omega)$, we define $\left(\hat{u}_{\varepsilon}, \hat{w}_{\varepsilon}, \hat{P}_{\varepsilon}\right)$ by

$$
\begin{gathered}
\hat{u}_{\varepsilon}\left(x^{\prime}, y\right)=\tilde{u}_{\varepsilon}\left(\varepsilon \kappa\left(\frac{x^{\prime}}{\varepsilon}\right)+\varepsilon y^{\prime}, y_{3}\right), \quad \hat{w}_{\varepsilon}\left(x^{\prime}, y\right)=\tilde{w}_{\varepsilon}\left(\varepsilon \kappa\left(\frac{x^{\prime}}{\varepsilon}\right)+\varepsilon y^{\prime}, y_{3}\right) \\
\hat{P}_{\varepsilon}\left(x^{\prime}, y\right)=\tilde{P}_{\varepsilon}\left(\varepsilon \kappa\left(\frac{x^{\prime}}{\varepsilon}\right)+\varepsilon y^{\prime}, y_{3}\right),
\end{gathered}
$$

a.e. $\left(x^{\prime}, y\right) \in \omega \times Y$, where $\tilde{u}_{\varepsilon}, \tilde{w}_{\varepsilon}$ and $\tilde{P}_{\varepsilon}$ are extended by zero outside $\Omega$ and the function $\kappa$ is defined by (2.4).

Remark 3.6. For $k^{\prime} \in \mathcal{K}_{\varepsilon}$, the restrictions of $\left(\hat{u}_{\varepsilon}, \hat{w}_{\varepsilon}, \hat{P}_{\varepsilon}\right)$ to $Y_{k^{\prime}, a_{\varepsilon}}^{\prime} \times Y$ does not depend on $x^{\prime}$, whereas as a function of $y$ it is obtained from $\left(\tilde{u}_{\varepsilon}, \tilde{w}_{\varepsilon}, \tilde{P}_{\varepsilon}\right)$ by using the change of variables $y^{\prime}=\frac{x^{\prime}-a_{\varepsilon} k^{\prime}}{a_{\varepsilon}}$, which transforms $Y_{k^{\prime}, a_{\varepsilon}}$ into $Y$.

Now, we get the estimates for the sequences $\left(\hat{u}_{\varepsilon}, \hat{w}_{\varepsilon}, \hat{P}_{\varepsilon}\right)$ similarly as in the proof of Lemma 4.9 in 4 .

Lemma 3.7. There exists a constant $C>0$ independent of $\varepsilon$, such that $\left(\hat{u}_{\varepsilon}, \hat{w}_{\varepsilon}, \hat{P}_{\varepsilon}\right)$ defined by (3.36)-(3.37) satisfies

i) in the cases PTMP and HTPM,

$$
\begin{aligned}
& \left\|\hat{u}_{\varepsilon}\right\|_{L^{2}(\omega \times Y)^{3}} \leq C a_{\varepsilon}^{2}, \quad\left\|D_{y^{\prime}} \hat{u}_{\varepsilon}\right\|_{L^{2}(\omega \times Y)^{3 \times 3}} \leq C a_{\varepsilon}^{2}, \quad\left\|\partial_{y_{3}} \hat{u}_{\varepsilon}\right\|_{L^{2}(\omega \times Y)^{3}} \leq C a_{\varepsilon} \varepsilon, \\
& \left\|\hat{w}_{\varepsilon}\right\|_{L^{2}(\omega \times Y)^{3}} \leq C a_{\varepsilon}, \quad\left\|D_{y^{\prime}} \hat{w}_{\varepsilon}\right\|_{L^{2}(\omega \times Y)^{3 \times 3}} \leq C a_{\varepsilon}, \quad\left\|\partial_{y_{3}} \hat{w}_{\varepsilon}\right\|_{L^{2}(\omega \times Y)^{3}} \leq C \varepsilon,
\end{aligned}
$$

ii) in the case VTPM,

$$
\begin{gathered}
\left\|\hat{u}_{\varepsilon}\right\|_{L^{2}(\omega \times Y)^{3}} \leq C \varepsilon^{2}, \quad\left\|D_{y^{\prime}} \hat{u}_{\varepsilon}\right\|_{L^{2}(\omega \times Y)^{3 \times 3}} \leq C a_{\varepsilon} \varepsilon, \quad\left\|\partial_{y_{3}} \hat{u}_{\varepsilon}\right\|_{L^{2}(\omega \times Y)^{3}} \leq C \varepsilon^{2}, \\
\left\|\hat{w}_{\varepsilon}\right\|_{L^{2}(\omega \times Y)^{3}} \leq C \varepsilon, \quad\left\|D_{y^{\prime}} \hat{w}_{\varepsilon}\right\|_{L^{2}(\omega \times Y)^{3 \times 3}} \leq C a_{\varepsilon}, \quad\left\|\partial_{y_{3}} \hat{w}_{\varepsilon}\right\|_{L^{2}(\omega \times Y)^{3}} \leq C \varepsilon,
\end{gathered}
$$

and, moreover, in every cases,

$$
\left\|\hat{P}_{\varepsilon}\right\|_{L^{2}(\omega \times Y)} \leq C
$$


Weak variational formulation. To finish this section, we will give the variational formulation satisfied by the functions $\left(\hat{u}_{\varepsilon}, \hat{w}_{\varepsilon}, \hat{P}_{\varepsilon}\right)$, which will be useful in the following sections.

We consider $\varphi_{\varepsilon}\left(x^{\prime}, y_{3}\right)=\varphi\left(x^{\prime}, x^{\prime} / \varepsilon, y_{3}\right)$ and $\psi_{\varepsilon}\left(x^{\prime}, y_{3}\right)=\psi\left(x^{\prime}, x^{\prime} / \varepsilon, y_{3}\right)$ as test function in (2.13) where $\varphi\left(x^{\prime}, y\right), \psi\left(x^{\prime}, y\right) \in \mathcal{D}\left(\omega ; C_{\#}^{\infty}(Y)^{3}\right)$, and taking into account the extension of the pressure, we have

$$
\int_{\widetilde{\Omega}_{\varepsilon}} \nabla_{\varepsilon} \tilde{p}_{\varepsilon} \cdot \varphi_{\varepsilon} d x^{\prime} d y_{3}=\int_{\Omega} \nabla_{\varepsilon} \tilde{P}_{\varepsilon} \cdot \varphi_{\varepsilon} d x^{\prime} d y_{3}
$$

and the extension of $\left(\tilde{u}_{\varepsilon}, \tilde{w}_{\varepsilon}\right)$, we get

$$
\left\{\begin{array}{l}
\int_{\widetilde{\Omega}_{\varepsilon}} D_{\varepsilon} \tilde{u}_{\varepsilon}: D_{\varepsilon} \varphi_{\varepsilon} d x^{\prime} d y_{3}-\int_{\Omega} \tilde{P}_{\varepsilon} \operatorname{div}_{\varepsilon} \varphi_{\varepsilon} d x^{\prime} d y_{3}=2 N^{2} \int_{\widetilde{\Omega}_{\varepsilon}} \operatorname{rot}_{\varepsilon} \tilde{w}_{\varepsilon} \cdot \varphi_{\varepsilon} d x^{\prime} d y_{3}+\int_{\widetilde{\Omega}_{\varepsilon}} f^{\prime} \cdot \varphi_{\varepsilon}^{\prime} d x^{\prime} d y_{3}, \\
R_{M} \int_{\widetilde{\Omega}_{\varepsilon}} D_{\varepsilon} \tilde{w}_{\varepsilon}: D_{\varepsilon} \psi_{\varepsilon} d x^{\prime} d y_{3}+4 N^{2} \int_{\widetilde{\Omega}_{\varepsilon}} \tilde{w}_{\varepsilon} \cdot \psi_{\varepsilon} d x^{\prime} d y_{3}=2 N^{2} \int_{\widetilde{\Omega}_{\varepsilon}} \operatorname{rot}_{\varepsilon} \tilde{u}_{\varepsilon} \cdot \psi_{\varepsilon} d x^{\prime} d y_{3}+\int_{\widetilde{\Omega}_{\varepsilon}} g_{\varepsilon}^{\prime} \cdot \psi_{\varepsilon}^{\prime} d x^{\prime} d y_{3},
\end{array}\right.
$$

where $R_{M}$ and $g_{\varepsilon}^{\prime}$ depend on the case, see assumptions (3.14)-(3.17).

Now, by the change of variables given in Remark 3.6] (see [4] for more details), we obtain

$$
\left\{\begin{array}{l}
\frac{1}{a_{\varepsilon}^{2}} \int_{\omega \times Y_{f}} D_{y^{\prime}} \hat{u}_{\varepsilon}^{\prime}: D_{y^{\prime}} \varphi^{\prime} d x^{\prime} d y+\frac{1}{\varepsilon^{2}} \int_{\omega \times Y_{f}} \partial_{y_{3}} \hat{u}_{\varepsilon}^{\prime}: \partial_{y_{3}} \varphi^{\prime} d x^{\prime} d y \\
-\int_{\omega \times Y_{f}} \hat{P}_{\varepsilon} \operatorname{div}_{x^{\prime}} \varphi^{\prime} d x^{\prime} d y-\frac{1}{a_{\varepsilon}} \int_{\omega \times Y_{f}} \hat{P}_{\varepsilon} \operatorname{div}_{y^{\prime}} \varphi^{\prime} d x^{\prime} d y \\
=\frac{2 N^{2}}{a_{\varepsilon}} \int_{\omega \times Y_{f}} \operatorname{rot}_{y^{\prime}} \hat{w}_{\varepsilon, 3} \cdot \varphi^{\prime} d x^{\prime} d y+\frac{2 N^{2}}{\varepsilon} \int_{\omega \times Y_{f}} \operatorname{rot}_{y_{3}} \hat{w}_{\varepsilon}^{\prime} \cdot \varphi^{\prime} d x^{\prime} d y+\int_{\omega \times Y_{f}} f^{\prime} \cdot \varphi^{\prime} d x^{\prime} d y+O_{\varepsilon}, \\
\frac{1}{a_{\varepsilon}^{2}} \int_{\omega \times Y_{f}} \nabla_{y^{\prime}} \hat{u}_{\varepsilon, 3} \cdot \nabla_{y^{\prime}} \varphi_{3} d x^{\prime} d y+\frac{1}{\varepsilon^{2}} \int_{\omega \times Y_{f}} \partial_{y_{3}} \hat{u}_{\varepsilon, 3} \cdot \partial_{y_{3}} \varphi_{3} d x^{\prime} d y-\frac{1}{\varepsilon} \int_{\omega \times Y_{f}} \hat{P}_{\varepsilon} \partial_{y_{3}} \varphi_{3} d x^{\prime} d y \\
=\frac{2 N^{2}}{a_{\varepsilon}} \int_{\omega \times Y_{f}} \operatorname{Rot}_{y^{\prime}} \hat{w}_{\varepsilon}^{\prime} \varphi_{3} d x^{\prime} d y+O_{\varepsilon},
\end{array}\right.
$$

and

$$
\left\{\begin{array}{l}
\frac{R_{M}}{a_{\varepsilon}^{2}} \int_{\omega \times Y_{f}} D_{y^{\prime}} \hat{w}_{\varepsilon}^{\prime}: D_{y^{\prime}} \psi^{\prime} d x^{\prime} d y+\frac{R_{M}}{\varepsilon^{2}} \int_{\omega \times Y_{f}} \partial_{y_{3}} \hat{w}_{\varepsilon}^{\prime}: \partial_{y_{3}} \psi^{\prime} d x^{\prime} d y+4 N^{2} \int_{\omega \times Y_{f}} \hat{w}_{\varepsilon}^{\prime} \cdot \psi^{\prime} d x^{\prime} d y \\
=\frac{2 N^{2}}{a_{\varepsilon}} \int_{\omega \times Y_{f}} \operatorname{rot}_{y^{\prime}} \hat{u}_{\varepsilon, 3} \cdot \psi^{\prime} d x^{\prime} d y+\frac{2 N^{2}}{\varepsilon} \int_{\omega \times Y_{f}} \operatorname{rot}_{y_{3}} \hat{u}_{\varepsilon}^{\prime} \cdot \psi^{\prime} d x^{\prime} d y+\int_{\omega \times Y_{f}} g_{\varepsilon}^{\prime} \cdot \psi^{\prime} d x^{\prime} d y+O_{\varepsilon}, \\
\frac{R_{M}}{a_{\varepsilon}^{2}} \int_{\omega \times Y_{f}} \nabla_{y^{\prime}} \hat{w}_{\varepsilon, 3} \cdot \nabla_{y^{\prime}} \psi_{3} d x^{\prime} d y+\frac{R_{M}}{\varepsilon^{2}} \int_{\omega \times Y_{f}} \partial_{y_{3}} \hat{w}_{\varepsilon, 3}: \partial_{y_{3}} \psi_{3} d x^{\prime} d y+4 N^{2} \int_{\omega \times Y_{f}} \hat{w}_{\varepsilon, 3} \cdot \psi_{3} d x^{\prime} d y \\
=\frac{2 N^{2}}{a_{\varepsilon}} \int_{\omega \times Y_{f}} \operatorname{Rot}_{y^{\prime}} \hat{u}_{\varepsilon}^{\prime} \psi_{3} d x^{\prime} d y+O_{\varepsilon} .
\end{array}\right.
$$

When $\varepsilon$ tends to zero, we obtain for $\left(\hat{u}_{\varepsilon}, \hat{w}_{\varepsilon}, \hat{P}_{\varepsilon}\right)$ different asymptotic behaviors depending on the cases PTPM, HTPM and VTPM. We will analyze them in the next sections.

\section{Proportionally Thin Porous Medium (PTPM)}

It corresponds to the critical case when the cylinder height is proportional to the interspatial distance, with $\lambda$ the proportionality constant, that is $a_{\varepsilon} \approx \varepsilon$, with $a_{\varepsilon} / \varepsilon \rightarrow \lambda, 0<\lambda<+\infty$. 
Let us introduce some notation which will be useful along this section. For a vectorial function $v=\left(v^{\prime}, v_{3}\right)$ and a scalar function $w$, we introduce the operators $D_{\lambda}, \nabla_{\lambda}, \operatorname{div}_{\lambda}$ and $\operatorname{rot}_{\lambda}$ by

$$
\begin{gathered}
\left(D_{\lambda} v\right)_{i j}=\partial_{x_{j}} v_{i} \text { for } i=1,2,3, j=1,2, \quad\left(D_{\lambda} v\right)_{i, 3}=\lambda \partial_{y_{3}} v_{i} \text { for } i=1,2,3, \\
\Delta_{\lambda} v=\Delta_{y^{\prime}} v+\lambda^{2} \partial_{y_{3}}^{2} v, \quad \nabla_{\lambda} w=\left(\nabla_{y^{\prime}} w, \lambda \partial_{y_{3}} w\right)^{t} \\
\operatorname{div}_{\lambda} v=\operatorname{div}_{y^{\prime}} v^{\prime}+\lambda \partial_{y_{3}} v_{3}, \quad \operatorname{rot}_{\lambda} v=\left(\operatorname{rot}_{y^{\prime}} v_{3}+\lambda \operatorname{rot}_{y_{3}} v^{\prime}, \operatorname{Rot}_{y^{\prime}} v^{\prime}\right)
\end{gathered}
$$

where $\operatorname{rot}_{y^{\prime}}, \operatorname{rot}_{y_{3}}$ and $\operatorname{Rot}_{y^{\prime}}$ are defined in (2.9). Next, we give some compactness results about the behavior of the extended sequences $\left(\tilde{u}_{\varepsilon}, \tilde{w}_{\varepsilon}, \tilde{P}_{\varepsilon}\right)$ and the unfolding functions $\left(\hat{u}_{\varepsilon}, \hat{w}_{\varepsilon}, \hat{P}_{\varepsilon}\right)$ satisfying the a priori estimates given in Lemmas 3.4 , 3.5 and 3.7 respectively.

Lemma 4.1. For a subsequence of $\varepsilon$ still denote by $\varepsilon$, we have that

i) (Velocity) there exist $\tilde{u} \in H_{0}^{1}\left(0,1 ; L^{2}(\omega)^{3}\right)$ with $\tilde{u}_{3}=0$ and $\hat{u} \in L^{2}\left(\omega ; H_{0, \#}^{1}(Y)\right)^{3}$ with $\hat{u}=0$ on $\omega \times Y_{s}$, such that $\int_{Y} \hat{u}\left(x^{\prime}, y\right) d y=\int_{0}^{1} \tilde{u}\left(x^{\prime}, y_{3}\right) d y_{3}$ with $\int_{Y} \hat{u}_{3} d y=0$ and moreover

$$
\begin{gathered}
a_{\varepsilon}^{-2} \tilde{u}_{\varepsilon} \rightarrow\left(\tilde{u}^{\prime}, 0\right) \text { in } H^{1}\left(0,1 ; L^{2}(\omega)^{3}\right), \quad a_{\varepsilon}^{-2} \hat{u}_{\varepsilon} \rightarrow \hat{u} \text { in } L^{2}\left(\omega ; H^{1}(Y)^{3}\right), \\
\operatorname{div}_{x^{\prime}}\left(\int_{0}^{1} \tilde{u}^{\prime}\left(x^{\prime}, y_{3}\right) d y_{3}\right)=0 \text { in } \omega, \quad\left(\int_{0}^{1} \tilde{u}^{\prime}\left(x^{\prime}, y_{3}\right) d y_{3}\right) \cdot n=0 \text { in } \partial \omega, \\
\operatorname{div}_{\lambda} \hat{u}=0 \text { in } \omega \times Y, \quad \operatorname{div}_{x^{\prime}}\left(\int_{Y} \hat{u}^{\prime}\left(x^{\prime}, y\right) d y\right)=0 \text { in } \omega, \quad\left(\int_{Y} \hat{u}^{\prime}\left(x^{\prime}, y\right) d y\right) \cdot n=0 \text { in } \partial \omega,
\end{gathered}
$$

ii) (Microrotation) there exist $\tilde{w} \in H_{0}^{1}\left(0,1 ; L^{2}(\omega)^{3}\right)$ with $\tilde{w}_{3}=0$ and $\hat{w} \in L^{2}\left(\omega ; H_{0, \#}^{1}(Y)\right)^{3}$ with $\hat{w}=0$ on $\omega \times Y_{s}$, such that $\int_{Y} \hat{w}\left(x^{\prime}, y\right) d y=\int_{0}^{1} \tilde{w}\left(x^{\prime}, y_{3}\right) d y_{3}$ with $\int_{Y} \hat{w}_{3} d y=0$ and moreover

$$
a_{\varepsilon}^{-1} \tilde{w}_{\varepsilon} \rightarrow\left(\tilde{w}^{\prime}, 0\right) \text { in } H^{1}\left(0,1 ; L^{2}(\omega)^{3}\right), \quad a_{\varepsilon}^{-1} \hat{w}_{\varepsilon} \rightarrow \hat{w} \text { in } L^{2}\left(\omega ; H^{1}(Y)^{3}\right)
$$

iii) (Pressure) there exists a function $\tilde{P} \in L_{0}^{2}(\Omega)$, independent of $y_{3}$, such that

$$
\tilde{P}_{\varepsilon} \rightarrow \tilde{P} \text { in } L^{2}(\Omega), \quad \hat{P}_{\varepsilon} \rightarrow \tilde{P} \text { in } L^{2}(\omega \times Y) .
$$

Proof. The proof of this result for the velocity is obtained by arguing similarly to Section 5 in [4].

The proof of the results for the microrotation is analogous to the ones of the velocity, except to prove that $\tilde{w}_{3}=0$. To do this, we consider as test function $\psi_{\varepsilon}\left(x^{\prime}, y_{3}\right)=\left(0,0, a_{\varepsilon}^{-1} \psi_{3}\right)$ in the variational formulation (3.42), and we get

$$
\begin{aligned}
& a_{\varepsilon} R_{c} \int_{\Omega} \nabla_{x^{\prime}} \tilde{w}_{\varepsilon, 3} \cdot \nabla_{x^{\prime}} \psi_{3} d x^{\prime} d y_{3}+a_{\varepsilon} \varepsilon^{-2} R_{c} \int_{\Omega} \partial_{y_{3}} \tilde{w}_{\varepsilon, 3} \partial_{y_{3}} \psi_{3} d x^{\prime} d y_{3}+4 N^{2} a_{\varepsilon}^{-1} \int_{\Omega} \tilde{w}_{\varepsilon, 3} \psi_{3} d x^{\prime} d y_{3} \\
& =2 N^{2} a_{\varepsilon}^{-1} \int_{\Omega} \operatorname{Rot}_{x^{\prime}} \tilde{u}_{\varepsilon}^{\prime} \psi_{3} d x^{\prime} d y_{3} .
\end{aligned}
$$

Passing to the limit by using convergences of $\tilde{u}_{\varepsilon}$ and $\tilde{w}_{\varepsilon}$ given in (4.45) and (4.48), we get

$$
\lambda^{2} R_{c} \int_{\Omega} \partial_{y_{3}} \tilde{w}_{3} \partial_{y_{3}} \psi_{3} d x^{\prime} d y_{3}+4 N^{2} \int_{\Omega} \tilde{w}_{3} \psi_{3} d x^{\prime} d y_{3}=0
$$

and taking into account that $\tilde{w}_{3}=0$ on $y_{3}=\{0,1\}$, it is easily deduced that $\tilde{w}_{3}=0$ a.e. in $\Omega$.

We finish with the proof for the pressure. Estimate (3.7) implies, up to a subsequence, the existence of $\tilde{P} \in L_{0}^{2}(\Omega)$ such that

$$
\tilde{P}_{\varepsilon} \rightarrow \tilde{P} \quad \text { in } L^{2}(\Omega) .
$$


Also, from $\left\|\nabla_{\varepsilon} \tilde{P}_{\varepsilon}\right\|_{L^{2}(\Omega)^{3}} \leq C$, by noting that $\partial_{y_{3}} \tilde{P}_{\varepsilon} / \varepsilon$ also converges weakly in $H^{-1}(\Omega)$, we obtain $\partial_{y_{3}} \tilde{P}=0$ and so $\tilde{P}$ is independent of $y_{3}$.

Next, following [22], we prove that the convergence of the pressure is in fact strong. As $\tilde{u}_{3}=0, \partial_{y_{3}} \tilde{P}_{\varepsilon}$ tends to zero and $\tilde{p}$ only depends on $x^{\prime}$, let $\sigma_{\varepsilon}\left(x^{\prime}, y_{3}\right)=\left(\sigma_{\varepsilon}^{\prime}\left(x^{\prime}\right), 0\right) \in H_{0}^{1}(\omega)^{3}$ be such that

$$
\sigma_{\varepsilon} \rightarrow \sigma \quad \text { in } H_{0}^{1}(\omega)^{3} .
$$

Then, we have

$$
\left|<\nabla_{x^{\prime}} \tilde{P}_{\varepsilon}, \sigma_{\varepsilon}>_{\Omega}-<\nabla_{x^{\prime}} \tilde{P}, \sigma>_{\Omega}\right| \leq\left|<\nabla_{x^{\prime}} \tilde{P}_{\varepsilon}, \sigma_{\varepsilon}-\sigma>_{\Omega}\right|+\left|<\nabla_{x^{\prime}} \tilde{P}_{\varepsilon}-\nabla_{x^{\prime}} \tilde{P}, \sigma>_{\Omega}\right| .
$$

On the one hand, using convergence (4.50), we have

$$
\left|<\nabla_{x^{\prime}} \tilde{P}_{\varepsilon}-\nabla_{x^{\prime}} \tilde{P}, \sigma>_{\Omega}\right|=\left|\int_{\Omega}\left(\tilde{P}_{\varepsilon}-\tilde{P}\right) \operatorname{div}_{x^{\prime}} \sigma^{\prime} d x\right| \rightarrow 0, \quad \text { as } \varepsilon \rightarrow 0 .
$$

On the other hand, from (3.34) and following the proof of Lemma 3.5, we have that

$$
\begin{aligned}
\left|<\nabla_{x^{\prime}} \tilde{P}_{\varepsilon}, \sigma_{\varepsilon}-\sigma>_{\Omega}\right|= & \left|<\nabla_{x^{\prime}} \tilde{P}_{\varepsilon}, \tilde{R}^{\varepsilon}\left(\sigma_{\varepsilon}^{\prime}-\sigma^{\prime}\right)>_{\tilde{\Omega}_{\varepsilon}}\right| \\
& \leq C\left(\left\|\sigma_{\varepsilon}^{\prime}-\sigma^{\prime}\right\|_{L^{2}(\omega)^{3}}+a_{\varepsilon}\left\|D_{x^{\prime}}\left(\sigma_{\varepsilon}^{\prime}-\sigma^{\prime}\right)\right\|_{L^{2}(\omega)^{3}}\right) \rightarrow 0 \quad \text { as } \varepsilon \rightarrow 0,
\end{aligned}
$$

by virtue of (4.51) and the Rellich theorem. This implies that $\nabla_{x^{\prime}} \tilde{P}_{\varepsilon} \rightarrow \nabla_{x^{\prime}} \tilde{P}$ strongly in $H^{-1}(\Omega)^{3}$, which together the Nečas inequality, implies the strong convergence of the pressure $\tilde{P}_{\varepsilon}$ given in (4.49). We finish the proof by proving that $\hat{P}_{\varepsilon}$ also converges strongly to $\tilde{P}$. The existence of $\hat{q} \in L^{2}(\omega \times Y)$ such that $\hat{P}_{\varepsilon}$ converges weakly to $\hat{q}$ is a consequence of estimate (3.7). It remains only to prove that $\hat{q}$ does not depend on the microscopic variable $y$. To do this, we choose as test function $\varphi_{\varepsilon}\left(x^{\prime}, y\right)=\left(\varepsilon \varphi^{\prime}, \eta_{\varepsilon} \varphi_{3}\right)$ in (3.43). Taking into account the estimates (3.38)-(3.7) and passing to the limit when $\varepsilon$ tends to zero by using convergence (4.49), we have

$$
\int_{\omega \times Y} \hat{P} \operatorname{div}_{y} \varphi d x^{\prime} d y=0
$$

which shows that $\hat{P}$ does not depend on $y$, which ends the proof.

Unsing previous convergences, in the following theorem we give the homogenized system satisfied by $(\hat{u}, \hat{w}, \tilde{P})$.

Theorem 4.2. In the case PTPM, the sequence $\left(a_{\varepsilon}^{-2} \hat{u}_{\varepsilon}, a_{\varepsilon}^{-1} \hat{w}_{\varepsilon}\right)$ converges weakly to $(\hat{u}, \hat{w})$ in $L^{2}\left(\omega ; H^{1}(Y)^{3}\right) \times$ $L^{2}\left(\omega ; H^{1}(Y)^{3}\right)$ and $\hat{P}_{\varepsilon}$ converges strongly to $\tilde{P}$ in $L^{2}(\omega)$, where $(\hat{u}, \hat{w}, \tilde{P}) \in L^{2}\left(\omega ; H_{0, \#}^{1}(Y)^{3}\right) \times L^{2}\left(\omega ; H_{0, \#}^{1}(Y)^{3}\right) \times$ $\left(L_{0}^{2}(\omega) \cap H^{1}(\omega)\right)$, with $\int_{Y} \hat{u}_{3} d y=\int_{Y} \hat{w}_{3} d y=0$, is the unique solution of the following homogenized system

$$
\left\{\begin{aligned}
-\Delta_{\lambda} \hat{u}+\nabla_{\lambda} \hat{q}=2 N^{2} \operatorname{rot}_{\lambda} \hat{w}+f^{\prime}\left(x^{\prime}\right)-\nabla_{x^{\prime}} \tilde{P}\left(x^{\prime}\right) & \text { in } \omega \times Y_{f}, \\
\operatorname{div}_{\lambda} \hat{u}=0 & \text { in } \omega \times Y_{f}, \\
-R_{c} \Delta_{\lambda} \hat{w}+4 N^{2} \hat{w}=2 N^{2} \operatorname{rot}_{\lambda} \hat{u}+g^{\prime}\left(x^{\prime}\right) & \text { in } \omega \times Y_{f}, \\
\hat{u}=\hat{w}=0 & \text { in } \omega \times Y_{s}, \\
\operatorname{div}_{x^{\prime}}\left(\int_{Y} \hat{u}^{\prime}\left(x^{\prime}, y\right) d y\right)=0 & \text { in } \omega \\
\left(\int_{Y} \hat{u}^{\prime}\left(x^{\prime}, y\right) d y\right) \cdot n=0 & \text { on } \partial \omega, \\
\hat{u}\left(x^{\prime}, y\right), \hat{w}\left(x^{\prime}, y\right), \hat{q}\left(x^{\prime}, y\right) \quad Y^{\prime}-\text { periodic. } &
\end{aligned}\right.
$$


Proof. For every $\varphi \in \mathcal{D}\left(\omega ; C_{\#}^{\infty}(Y)^{3}\right)$ with $\operatorname{div}_{\lambda} \varphi=0$ in $\omega \times Y$ and $\operatorname{div}_{x^{\prime}}\left(\int_{Y} \varphi^{\prime} d y\right)=0$ in $\omega$, we choose $\varphi_{\varepsilon}=\left(\varphi^{\prime}, \lambda\left(\varepsilon / a_{\varepsilon}\right) \varphi_{3}\right)$ in (3.43). Taking into account that thanks to $\operatorname{div}_{\lambda} \varphi=0$ in $\omega \times Y$, we have that

$$
\frac{1}{a_{\varepsilon}} \int_{\omega \times Y} \hat{P}_{\varepsilon}\left(\operatorname{div}_{y^{\prime}} \varphi^{\prime}+\lambda \partial_{y_{3}} \varphi_{3}\right) d x^{\prime} d y=0 .
$$

Thus, passing to the limit using the convergences (4.45), (4.48), (4.49) and $\lambda\left(\varepsilon / \eta_{\varepsilon}\right) \rightarrow 1$, we obtain

$$
\begin{aligned}
& \int_{\omega \times Y_{f}} D_{\lambda} \hat{u}: D_{\lambda} \varphi d x^{\prime} d y-\int_{\omega \times Y} \tilde{P} \operatorname{div}_{x^{\prime}} \varphi^{\prime} d x^{\prime} d y \\
& =2 N^{2} \int_{\omega \times Y_{f}}\left(\operatorname{rot}_{y^{\prime}} \hat{w}_{3} \cdot \varphi^{\prime}+\lambda \operatorname{rot}_{y_{3}} \hat{w}^{\prime} \cdot \varphi^{\prime}+\operatorname{Rot}_{y^{\prime}} \hat{w}^{\prime} \varphi_{3}\right) d x^{\prime} d y+\int_{\omega \times Y_{f}} f^{\prime} \cdot \varphi^{\prime} d x^{\prime} d y .
\end{aligned}
$$

Since $\tilde{P}$ does not depend on $y$ and $\operatorname{div}_{x^{\prime}} \int_{Y} \varphi^{\prime} d y=0$ in $\omega$, we have that the second term is zero, and so we get

$$
\int_{\omega \times Y_{f}} D_{\lambda} \hat{u}: D_{\lambda} \varphi d x^{\prime} d y=2 N^{2} \int_{\omega \times Y_{f}} \operatorname{rot}_{\lambda} \hat{w} \cdot \varphi d x^{\prime} d y+\int_{\omega \times Y_{f}} f^{\prime} \cdot \varphi^{\prime} d x^{\prime} d y .
$$

Next, for every $\psi \in \mathcal{D}\left(\omega ; C_{\#}^{\infty}(Y)^{3}\right)$, we choose $\psi_{\varepsilon}=a_{\varepsilon}^{-1} \psi$ in (3.44) with $g_{\varepsilon}$ and $R_{M}$ satisfying (3.14) and (3.15). Then, passing to the limit using convergences (4.45) and (4.48), we get

$$
R_{c} \int_{\omega \times Y_{f}} D_{\lambda} \hat{w}: D_{\lambda} \psi d x^{\prime} d y+4 N^{2} \int_{\omega \times Y_{f}} \hat{w} \cdot \psi d x^{\prime} d y=2 N^{2} \int_{\omega \times Y_{f}} \operatorname{rot}_{\lambda} \hat{u} \cdot \psi d x^{\prime} d y+\int_{\omega \times Y_{f}} g^{\prime} \cdot \psi^{\prime} d x^{\prime} d y .
$$

By density (4.54) holds for every function $\varphi$ in the Hilbert space $V$ defined by

$$
V=\left\{\begin{array}{l}
\varphi\left(x^{\prime}, y\right) \in L^{2}\left(\omega ; H_{0, \#}^{1}(Y)^{3}\right) \text { such that } \\
\operatorname{div}_{x^{\prime}}\left(\int_{Y_{f}} \varphi\left(x^{\prime}, y\right) d y\right)=0 \text { in } \omega, \quad\left(\int_{Y_{f}} \varphi\left(x^{\prime}, y\right) d y\right) \cdot n=0 \text { on } \partial \omega \\
\operatorname{div}_{\lambda} \varphi\left(x^{\prime}, y\right)=0 \text { in } \omega \times Y_{f}, \quad \varphi\left(x^{\prime}, y\right)=0 \text { in } \omega \times Y_{s}
\end{array}\right\},
$$

and (4.55) in $W=\left\{\psi\left(x^{\prime}, y\right) \in L^{2}\left(\omega ; H_{0, \#}^{1}(Y)^{3}\right): \psi\left(x^{\prime}, y\right)=0\right.$ in $\left.\omega \times Y_{s}\right\}$.

From Theorem 2.4.2 in [17, the variational formulation (4.54)-(4.55) admits a unique solution $(\hat{u}, \hat{w})$ in $V \times W$.

From Lemma 2.4.1 in [17] (see also [1), the orthogonal of $V$ with respect to the usual scalar product in $L^{2}(\omega \times Y)$ is made of gradients of the form $\nabla_{x^{\prime}} q\left(x^{\prime}\right)+\nabla_{\lambda} \hat{q}\left(x^{\prime}, y\right)$, with $q\left(x^{\prime}\right) \in L_{0}^{2}(\omega)$ and $\hat{q}\left(x^{\prime}, y\right) \in L^{2}\left(\omega ; H_{\#}^{1}(Y)\right)$. Therefore, by integration by parts, the variational formulations (4.54)-(4.55) are equivalent to the homogenized system (4.52). It remains to prove that the pressure $\tilde{P}\left(x^{\prime}\right)$, arising as a Lagrange multiplier of the incompressibility constraint $\operatorname{div}_{x^{\prime}}\left(\int_{Y} \hat{u}\left(x^{\prime}, y\right) d y\right)=0$, is the same as the limit of the pressure $\hat{P}_{\varepsilon}$. This can be easily done by considering in equation (3.43) a test function with $\operatorname{div}_{\lambda}$ equal to zero, and obtain the variational formulation (4.53). Since $2 N^{2} \operatorname{rot}_{\lambda} \hat{w}+f^{\prime} \in L^{2}(\omega \times Y)^{3}$ we deduce that $\tilde{P} \in H^{1}(\omega)$.

Finally, since from Lemma 2.4.1 in [17] we have that (4.52) admits a unique solution, and then the complete sequence $\left(a_{\varepsilon}^{-2} \hat{u}_{\varepsilon}, a_{\varepsilon}^{-1} \hat{w}_{\varepsilon}, \hat{P}_{\varepsilon}\right)$ converges to the solution $\left(\hat{u}\left(x^{\prime}, y\right), \hat{w}\left(x^{\prime}, y\right), \tilde{P}\left(x^{\prime}\right)\right)$.

Let us define the local problems which are useful to eliminate the variable $y$ of the previous homogenized problem and then obtain a Darcy equation for the pressure $\tilde{P}$. 
For every $i, k=1,2$ and $0<\lambda<+\infty$, we consider the following local micropolar problems

$$
\left\{\begin{aligned}
-\Delta_{\lambda} u^{i, k}+\nabla_{\lambda} \pi^{i, k}-2 N^{2} \operatorname{rot}_{\lambda} w^{i, k}=e_{i} \delta_{1 k} & \text { in } Y_{f}, \\
\operatorname{div}_{\lambda} u^{i, k}=0 & \text { in } Y_{f}, \\
-R_{c} \Delta_{\lambda} w^{i, k}+4 N^{2} w^{i, k}-2 N^{2} \operatorname{rot}_{\lambda} u^{i, k}=e_{i} \delta_{2 k} & \text { in } Y_{f}, \\
u^{i, k}=w^{i, k}=0 & \text { in } \omega \times Y_{s}, \\
\int_{Y_{f}} u_{3}^{i, k}(y) d y=\int_{Y_{f}} w_{3}^{i, k}(y) d y=0 & \text { in } \omega, \\
u^{i, k}(y), w^{i, k}(y), \pi^{i, k}(y) \quad Y^{\prime} \text { - periodic. } &
\end{aligned}\right.
$$

It is known (see Lemma 2.5.1 in [17]) that there exist a unique solution $\left(u^{i, k}, w^{i, k}, \pi^{i, k}\right) \in H_{0, \#}^{1}\left(Y_{f}\right)^{3} \times H_{0, \#}^{1}\left(Y_{f}\right)^{3} \times$ $L_{0}^{2}\left(Y_{f}\right)$ of problem (4.56), and moreover $\pi^{i, k} \in H^{1}\left(Y_{f}\right)$.

Now, we give the main result concerning the homogenized flow.

Theorem 4.3. Let $(\hat{u}, \hat{w}, \tilde{P}) \in L^{2}\left(\omega ; H_{0, \#}^{1}(Y)^{3}\right) \times L^{2}\left(\omega ; H_{0, \#}^{1}(Y)^{3}\right) \times\left(L_{0}^{2}(\omega) \cap H^{1}(\omega)\right)$ be the unique weak solution of problem 4.52). Then, the extensions $\left(a_{\varepsilon}^{-2} \tilde{u}_{\varepsilon}, a_{\varepsilon}^{-1} \tilde{w}_{\varepsilon}\right)$ and $\tilde{P}_{\varepsilon}$ of the solution of problem (2.10)- (2.11) converge weakly to $(\tilde{u}, \tilde{w})$ in $H^{1}\left(0,1 ; L^{2}(\omega)^{3}\right) \times H^{1}\left(0,1 ; L^{2}(\omega)^{3}\right)$ and strongly to $\tilde{P}$ in $L^{2}(\omega)$ respectively, with $\tilde{u}_{3}=\tilde{w}_{3}=0$. Moreover, defining $\widetilde{U}\left(x^{\prime}\right)=\int_{0}^{1} \tilde{u}\left(x^{\prime}, y_{3}\right) d y_{3}$ and $\widetilde{W}\left(x^{\prime}\right)=\int_{0}^{1} \tilde{w}\left(x^{\prime}, y_{3}\right) d y_{3}$, it holds

$$
\begin{array}{ll}
\widetilde{U}^{\prime}\left(x^{\prime}\right)=K_{\lambda}^{(1)}\left(f^{\prime}\left(x^{\prime}\right)-\nabla_{x^{\prime}} \tilde{P}\left(x^{\prime}\right)\right)+K_{\lambda}^{(2)} g\left(x^{\prime}\right), \quad \widetilde{U}_{3}\left(x^{\prime}\right)=0 \quad \text { in } \omega, \\
\widetilde{W^{\prime}}\left(x^{\prime}\right)=L_{\lambda}^{(1)}\left(f^{\prime}\left(x^{\prime}\right)-\nabla_{x^{\prime}} \tilde{P}\left(x^{\prime}\right)\right)+L_{\lambda}^{(2)} g\left(x^{\prime}\right), \quad \widetilde{W}_{3}\left(x^{\prime}\right)=0 \quad \text { in } \omega,
\end{array}
$$

where $K_{\lambda}^{(k)}, L_{\lambda}^{(k)} \in \mathbb{R}^{2 \times 2}, k=1,2$, are matrices with coefficients

$$
\left(K_{\lambda}^{(k)}\right)_{i j}=\int_{Y_{f}} u_{j}^{i, k}(y) d y, \quad\left(L_{\lambda}^{(k)}\right)_{i j}=\int_{Y_{f}} w_{j}^{i, k}(y) d y, \quad i, j=1,2
$$

where $u^{i, k}, w^{i, k}$ are the solutions of the local micropolar problems defined in (4.56).

Here, $\tilde{P} \in H^{1}(\omega) \cap L_{0}^{2}(\omega)$ is the unique solution of the $2 D$ Darcy equation

$$
\left\{\begin{array}{l}
\operatorname{div}_{x^{\prime}}\left(K_{\lambda}^{(1)}\left(f^{\prime}\left(x^{\prime}\right)-\nabla_{x^{\prime}} \tilde{P}\left(x^{\prime}\right)\right)+K_{\lambda}^{(2)} g\left(x^{\prime}\right)\right)=0 \quad \text { in } \omega \\
\left(K_{\lambda}^{(1)}\left(f^{\prime}\left(x^{\prime}\right)-\nabla_{x^{\prime}} \tilde{P}\left(x^{\prime}\right)\right)+K_{\lambda}^{(2)} g\left(x^{\prime}\right)\right) \cdot n=0 \quad \text { in } \partial \omega
\end{array}\right.
$$

Proof. We eliminate the microscopic variable $y$ in the effective problem (4.52). To do that, we consider the following identification

$$
\begin{gathered}
\hat{u}\left(x^{\prime}, y\right)=\sum_{i=1}^{2}\left[\left(f_{i}\left(x^{\prime}\right)-\partial_{x_{i}} \tilde{P}\left(x^{\prime}\right)\right) u^{i, 1}(y)+g_{i}\left(x^{\prime}\right) u^{i, 2}(y)\right], \\
\hat{w}\left(x^{\prime}, y\right)=\sum_{i=1}^{2}\left[\left(f_{i}\left(x^{\prime}\right)-\partial_{x_{i}} \tilde{P}\left(x^{\prime}\right)\right) w^{i, 1}(y)+g_{i}\left(x^{\prime}\right) w^{i, 2}(y)\right], \\
\hat{q}\left(x^{\prime}, y\right)=\sum_{i=1}^{2}\left[\left(f_{i}\left(x^{\prime}\right)-\partial_{x_{i}} \tilde{P}\left(x^{\prime}\right)\right) \pi^{i, 1}(y)+g_{i}\left(x^{\prime}\right) \pi^{i, 2}(y)\right],
\end{gathered}
$$

and thanks to the identity $\int_{Y_{f}} \hat{\varphi}\left(x^{\prime}, y\right) d y=\int_{0}^{1} \tilde{\varphi}\left(x^{\prime}, y_{3}\right) d y_{3}$ with $\int_{Y_{f}} \hat{\varphi}_{3} d y=0$ satisfied by velocity and microrotation given in Lemma 4.1] we deduce that $\widetilde{U}$ and $\widetilde{W}$ are given by (4.57). 
Finally, the divergence condition with respect to the variable $x^{\prime}$ given in (4.52) together with the expression of $\widetilde{U}^{\prime}\left(x^{\prime}\right)$ gives (4.58), which has a unique solution since $K_{\lambda}^{(1)}$ is positive definite and then the whole sequence converges, see Part III - Theorem 2.5.2 in [17.

Remark 4.4. We observe that when $N$ is identically zero, taking into account the linear momentum equations from (4.52), we can deduce that the Darcy equation [4.58) agrees with the ones obtained in [5, 14] in the case PTPM.

\section{The homogeneously thin porous medium (HTPM)}

It corresponds to the case when the cylinder height is much larger than interspatial distance, i.e. $a_{\varepsilon} \ll \varepsilon$ which is equivalent to $\lambda=0$.

Next, we give some compactness results about the behavior of the extended sequences $\left(\tilde{u}_{\varepsilon}, \tilde{w}_{\varepsilon}, \tilde{P}_{\varepsilon}\right)$ and the unfolding functions $\left(\hat{u}_{\varepsilon}, \hat{w}_{\varepsilon}, \hat{P}_{\varepsilon}\right)$ by using the a priori estimates given in Lemmas 3.4 and 3.5 , and Lemma 3.7 . respectively.

Lemma 5.1. For a subsequence of $\varepsilon$ still denoted by $\varepsilon$, there exist the following functions:

i) (Velocity) there exist $\tilde{u} \in L^{2}(\Omega)^{3}$, with $\tilde{u}_{3}=0$ and $\hat{u} \in L^{2}\left(\Omega ; H_{\#}^{1}\left(Y^{\prime}\right)^{3}\right)$ with $\hat{u}=0$ on $\omega \times Y_{s}$, such that $\int_{Y_{f}} \hat{u}\left(x^{\prime}, y\right) d y=\int_{0}^{1} \tilde{u}\left(x^{\prime}, y_{3}\right) d y_{3}$ with $\int_{Y_{f}} \hat{u}_{3}\left(x^{\prime}, y\right) d y=0, \hat{u}_{3}$ independent of $y_{3}$ and moreover

$$
\begin{gathered}
a_{\varepsilon}^{-2} \tilde{u}_{\varepsilon} \rightarrow\left(\tilde{u}^{\prime}, 0\right) \text { in } H^{1}\left(0,1 ; L^{2}(\omega)^{3}\right), \quad a_{\varepsilon}^{-2} \hat{u}_{\varepsilon} \rightarrow \hat{u} \text { in } L^{2}\left(\Omega ; H^{1}\left(Y^{\prime}\right)^{3}\right) \\
\operatorname{div}_{x^{\prime}}\left(\int_{0}^{1} \tilde{u}^{\prime}\left(x^{\prime}, y_{3}\right) d y_{3}\right)=0 \text { in } \omega, \quad\left(\int_{0}^{1} \tilde{u}^{\prime}\left(x^{\prime}, y_{3}\right) d y_{3}\right) \cdot n=0 \text { in } \partial \omega \\
\operatorname{div}_{y^{\prime}} \hat{u}^{\prime}=0 \text { in } \omega \times Y_{f}, \quad \operatorname{div}_{x^{\prime}}\left(\int_{Y_{f}} \hat{u}^{\prime}\left(x^{\prime}, y\right) d y\right)=0 \text { in } \omega, \quad\left(\int_{Y_{f}} \hat{u}\left(x^{\prime}, y\right) d y\right) \cdot n=0 \text { in } \partial \omega,
\end{gathered}
$$

ii) (Microrotation) there exist $\tilde{w} \in L^{2}(\Omega)^{3}$ with $\tilde{w}_{3}=0$ and $\hat{w} \in L^{2}\left(\Omega ; H_{\#}^{1}\left(Y^{\prime}\right)^{3}\right)$ with $\hat{w}=0$ on $\omega \times Y_{s}$, such that $\int_{Y_{f}} \hat{w}\left(x^{\prime}, y\right) d y=\int_{0}^{1} \tilde{w}\left(x^{\prime}, y_{3}\right) d y_{3}$ with $\int_{Y_{f}} \hat{w}_{3}\left(x^{\prime}, y\right) d y=0, \hat{u}_{3}$ independent of $y_{3}$ and moreover

$$
a_{\varepsilon}^{-1} \tilde{w}_{\varepsilon} \rightarrow\left(\tilde{w}^{\prime}, 0\right) \text { in } H^{1}\left(0,1 ; L^{2}(\omega)^{3}\right), \quad a_{\varepsilon}^{-1} \hat{w}_{\varepsilon} \rightarrow \hat{w} \text { in } L^{2}\left(\Omega ; H^{1}\left(Y^{\prime}\right)^{3}\right),
$$

iii) (Pressure) there exists $\tilde{P} \in L_{0}^{2}(\Omega)$ independent of $y_{3}$, such that

$$
\tilde{P}_{\varepsilon} \rightarrow \tilde{P} \text { in } L^{2}(\omega), \quad \hat{P}_{\varepsilon} \rightarrow \tilde{P} \text { in } L^{2}(\omega) .
$$

Proof. The proof of this result is obtained by arguing similarly to Section 5 in [4] and Lemma 4.1 of the present paper.

Using previous convergences, in the following theorem we give the homogenized system satisfied by $(\hat{u}, \hat{w}, \tilde{P})$.

Theorem 5.2. In the case HTPM, the sequence $\left(a_{\varepsilon}^{-2} \hat{u}_{\varepsilon}, a_{\varepsilon}^{-1} \hat{w}_{\varepsilon}\right)$ converges weakly to $(\hat{u}, \hat{w})$ in $L^{2}\left(\Omega ; H^{1}\left(Y^{\prime}\right)^{3}\right) \times$ $L^{2}\left(\Omega ; H^{1}\left(Y^{\prime}\right)^{3}\right)$ and $\hat{P}_{\varepsilon}$ converges strongly to $\tilde{P}$ in $L^{2}(\omega)$, where $(\hat{u}, \hat{w}, \tilde{P}) \in L^{2}\left(\Omega ; H_{\#}^{1}\left(Y^{\prime}\right)^{3}\right) \times L^{2}\left(\Omega ; H_{\#}^{1}\left(Y^{\prime}\right)^{3}\right) \times$ $\left(L_{0}^{2}(\omega) \cap H^{1}(\omega)\right)$ with $\hat{u}_{3}$ and $\hat{w}_{3}$ independent of $y_{3}$ and $\int_{Y_{f}^{\prime}} \hat{u}_{3}\left(x^{\prime}, y^{\prime}\right) d y^{\prime}=\int_{Y_{f}^{\prime}} \hat{w}_{3}\left(x^{\prime}, y^{\prime}\right) d y^{\prime}=0$. Moreover, defining $\hat{U}=\int_{0}^{1} \hat{u}\left(x^{\prime}, y\right) d y_{3}, \hat{W}=\int_{0}^{1} \hat{u}\left(x^{\prime}, y\right) d y_{3}$, we have that $(\hat{U}, \hat{W}) \in L^{2}\left(\omega ; H_{\#}^{1}\left(Y^{\prime}\right)^{3}\right) \times L^{2}\left(\omega ; H_{\#}^{1}\left(Y^{\prime}\right)^{3}\right)$ is 
the unique solution of the following homogenized system

$$
\left\{\begin{array}{rc}
-\Delta_{y^{\prime}} \hat{W}^{\prime}+\nabla_{y^{\prime}} \hat{q}=2 N^{2} \operatorname{rot}_{y^{\prime}} \hat{W}_{3}+f^{\prime}\left(x^{\prime}\right)-\nabla_{x^{\prime}} \tilde{P}\left(x^{\prime}\right) & \text { in } \omega \times Y_{f}^{\prime}, \\
-\Delta_{y^{\prime}} \hat{W}_{3}=2 N^{2} \operatorname{Rot}_{y^{\prime}} \hat{W}^{\prime} & \text { in } \omega \times Y_{f}^{\prime}, \\
-R_{c} \Delta_{y^{\prime}} \hat{W}^{\prime}+4 N^{2} \hat{W}^{\prime}=2 N^{2} \operatorname{rot}_{y^{\prime}} \hat{U}_{3}+g^{\prime}\left(x^{\prime}\right) & \text { in } \omega \times Y_{f}^{\prime}, \\
-R_{c} \Delta_{y^{\prime}} \hat{W}_{3}+4 N^{2} \hat{W}_{3}=2 N^{2} \operatorname{Rot}_{y^{\prime}} \hat{U}^{\prime} & \text { in } \omega \times Y_{f}^{\prime}, \\
\operatorname{div}_{y^{\prime}} \hat{U}^{\prime}=0 & \text { in } \omega \times Y_{f}^{\prime}, \\
\hat{U}^{\prime}=\hat{W}^{\prime}=0 & \text { in } \omega \times Y_{s}^{\prime}, \\
\operatorname{div}_{x^{\prime}}\left(\int_{Y_{f}^{\prime}} \hat{U}^{\prime}\left(x^{\prime}, y^{\prime}\right) d y^{\prime}\right)=0 & \text { in } \omega, \\
\left(\int_{\left.Y_{f}^{\prime} \hat{U}^{\prime}\left(x^{\prime}, y^{\prime}\right) d y^{\prime}\right) \cdot n=0}\right. & \text { on } \partial \omega, \\
\hat{U}\left(x^{\prime}, y^{\prime}\right), \hat{w}\left(x^{\prime}, y^{\prime}\right), \hat{q}\left(x^{\prime}, y^{\prime}\right) \quad Y^{\prime}-\text { periodic. } &
\end{array}\right.
$$

Proof. We choose $\varphi \in \mathcal{D}\left(\omega ; C_{\#}^{\infty}(Y)^{3}\right)$ with $\operatorname{div}_{y^{\prime}} \varphi^{\prime}=0$ in $\omega \times Y$, $\operatorname{div}_{x^{\prime}}\left(\int_{Y} \varphi^{\prime} d y\right)=0$ in $\omega$ and $\varphi_{3}$ independent of $y_{3}$ in (3.43). Taking into account that thanks to $\operatorname{div}_{y^{\prime}} \varphi^{\prime}=0$ in $\omega \times Y_{f}$ and $\varphi_{3}$ independent of $y_{3}$, we have that

$$
\frac{1}{a_{\varepsilon}} \int_{\omega \times Y} \hat{P}_{\varepsilon} \operatorname{div}_{y^{\prime}} \varphi^{\prime} d x^{\prime} d y=0 \quad \text { and } \quad \frac{1}{\varepsilon} \int_{\omega \times Y} \partial_{y_{3}} \hat{P}_{\varepsilon} \partial_{y_{3}} \varphi_{3} d x^{\prime} d y=0 .
$$

Thus, passing to the limit using the convergences (5.60), (5.63), (5.64), $a_{\varepsilon} / \varepsilon \rightarrow 0$ and using in the limit that $\tilde{P}$ does not depend on $y$ and $\operatorname{div}_{x^{\prime}}\left(\int_{Y} \varphi^{\prime} d y\right)=0$, we obtain

$$
\left\{\begin{array}{l}
\int_{\omega \times Y_{f}} D_{y^{\prime}} \hat{u}^{\prime}: D_{y^{\prime}} \varphi^{\prime} d x^{\prime} d y=2 N^{2} \int_{\omega \times Y_{f}} \operatorname{rot}_{y^{\prime}} \hat{w}_{3} \cdot \varphi^{\prime} d x^{\prime} d y+\int_{\omega \times Y_{f}} f^{\prime} \cdot \varphi^{\prime} d x^{\prime} d y \\
\int_{\omega \times Y_{f}} \nabla_{y^{\prime}} \hat{u}_{3}: \nabla_{y^{\prime}} \varphi_{3} d x^{\prime} d y=2 N^{2} \int_{\omega \times Y_{f}} \operatorname{Rot}_{y^{\prime}} \hat{w}^{\prime} \varphi_{3} d x^{\prime} d y .
\end{array}\right.
$$

Next, for every $\psi \in \mathcal{D}\left(\omega ; C_{\#}^{\infty}(Y)^{3}\right)$ with $\psi_{3}$ independent of $y_{3}$, we choose $\psi_{\varepsilon}=a_{\varepsilon}^{-1} \psi$ in (3.44) taking into account that $g_{\varepsilon}$ and $R_{M}$ satisfy (3.14) and (3.15). Then, passing to the limit using convergences (5.60) and (5.63), we get

$$
\left\{\begin{array}{l}
R_{c} \int_{\omega \times Y_{f}} D_{y^{\prime}} \hat{w}^{\prime}: D_{y^{\prime}} \psi^{\prime} d x^{\prime} d y+4 N^{2} \int_{\omega \times Y_{f}} \hat{w}^{\prime} \cdot \psi^{\prime} d x^{\prime} d y=2 N^{2} \int_{\omega \times Y_{f}} \operatorname{rot}_{y^{\prime}} \hat{u}_{3} \cdot \psi^{\prime} d x^{\prime} d y+\int_{\omega \times Y_{f}} g^{\prime}\left(x^{\prime}\right) \cdot \psi^{\prime} d x^{\prime} d y \\
R_{c} \int_{\omega \times Y_{f}} \nabla_{y^{\prime}} \hat{w}_{3}: \nabla_{y^{\prime}} \psi_{3} d x^{\prime} d y+4 N^{2} \int_{\omega \times Y_{f}} \hat{w}_{3} \cdot \psi_{3} d x^{\prime} d y=2 N^{2} \int_{\omega \times Y_{f}} \operatorname{Rot}_{y^{\prime}} \hat{u}^{\prime} \cdot \psi_{3} d x^{\prime} d y .
\end{array}\right.
$$

We take into account that there is no $y_{3}$-dependence in the obtained variational formulation. For that, we can consider $\varphi, \psi$ independent of $y_{3}$, which implies that $(\hat{U}, \hat{W})$ satisfies the same variational formulation with integral in $\omega \times Y_{f}^{\prime}$. By density, we can deduce that the variational formulation for $(\hat{U}, \hat{W})$ is equivalent to problem (5.65).

The local problems to eliminate the variable $y$ of the previous homogenized problem can be defined by using the local system (4.56) with $\lambda=0$. In that case, since there is no $y_{3}$ dependence, then it is correct to consider all equations in 2D domain $Y_{f}^{\prime}$ instead of $Y_{f}$. Therefore, for every $i, k=1,2$, we consider $\left(u^{i, k}, w^{i, k}, \pi^{i, k}\right) \in$ 
$H^{1}\left(Y_{f}^{\prime}\right)^{3} \times H^{1}\left(Y_{f}^{\prime}\right)^{3} \times\left(H^{1}\left(Y_{f}^{\prime}\right) \cap L_{0}^{2}\left(Y_{f}^{\prime}\right)\right)$ the unique solutions of the following local micropolar problems

$$
\left\{\begin{array}{rc}
-\Delta_{y^{\prime}}\left(u^{i, k}\right)^{\prime}+\nabla_{y^{\prime}} \pi^{i, k}-2 N^{2} \operatorname{rot}_{y^{\prime}} w_{3}^{i, k}=e_{i} \delta_{1 k} & \text { in } Y_{f}^{\prime}, \\
-\Delta_{y^{\prime}} u_{3}^{i, k}-2 N^{2} \operatorname{Rot}_{y^{\prime}}\left(w^{i, k}\right)^{\prime}=0 & \text { in } Y_{f}^{\prime}, \\
\operatorname{div}_{y^{\prime}} u^{i, k}=0 & \text { in } Y_{f}, \\
-R_{c} \Delta_{y^{\prime}}\left(w^{i, k}\right)^{\prime}+4 N^{2}\left(w^{i, k}\right)^{\prime}-2 N^{2} \operatorname{rot}_{y^{\prime}} u_{3}^{i, k}=e_{i} \delta_{2 k} & \text { in } Y_{f}^{\prime}, \\
-R_{c} \Delta_{y^{\prime}} w_{3}^{i, k}+4 N^{2} w_{3}^{i, k}-2 N^{2} \operatorname{rot}_{y^{\prime}}\left(u^{i, k}\right)^{\prime}=0 & \text { in } Y_{f}^{\prime}, \\
u^{i, k}=w^{i, k}=0 & \text { in } Y_{s}^{\prime}, \\
\int_{Y_{f}^{\prime}} u_{3}^{i, k} d y^{\prime}=\int_{Y_{f}} w_{3}^{i, k} d y^{\prime}=0, & \\
u^{i, k}\left(y^{\prime}\right), w^{i, k}\left(y^{\prime}\right), \pi^{i, k}\left(y^{\prime}\right) & Y^{\prime}-\text { periodic. }
\end{array}\right.
$$

We give the main result concerning the homogenized flow.

Theorem 5.3. Let $(\hat{U}, \hat{W}, \tilde{P}) \in L^{2}\left(\omega ; H_{\#}^{1}\left(Y^{\prime}\right)^{3}\right) \times L^{2}\left(\omega ; H_{\#}^{1}\left(Y^{\prime}\right)^{3}\right) \times\left(L_{0}^{2}(\omega) \cap H^{1}(\omega)\right)$ be the unique weak solution of problem (5.65). Then, the extensions $\left(a_{\varepsilon}^{-2} \tilde{u}_{\varepsilon}, a_{\varepsilon}^{-1} \tilde{w}_{\varepsilon}\right)$ and $\tilde{P}_{\varepsilon}$ of the solution of problem (2.10)-(2.11) converge weakly to $(\tilde{u}, \tilde{w})$ in $L^{2}(\Omega)^{3} \times L^{2}(\Omega)^{3}$ and strongly to $\tilde{P}$ in $L^{2}(\omega)$ respectively, with $\tilde{u}_{3}=\tilde{w}_{3}=0$. Moreover, defining $\widetilde{U}\left(x^{\prime}\right)=\int_{0}^{1} \tilde{u}\left(x^{\prime}, y_{3}\right) d y_{3}$ and $\widetilde{W}\left(x^{\prime}\right)=\int_{0}^{1} \tilde{w}\left(x^{\prime}, y_{3}\right) d y_{3}$, it holds

$$
\begin{array}{lll}
\widetilde{U}^{\prime}\left(x^{\prime}\right)=K_{0}^{(1)}\left(f^{\prime}\left(x^{\prime}\right)-\nabla_{x^{\prime}} \tilde{P}\left(x^{\prime}\right)\right)+K_{0}^{(2)} g\left(x^{\prime}\right), & \widetilde{U}_{3}\left(x^{\prime}\right)=0 & \text { in } \omega, \\
\widetilde{W}^{\prime}\left(x^{\prime}\right)=L_{0}^{(1)}\left(f^{\prime}\left(x^{\prime}\right)-\nabla_{x^{\prime}} \tilde{P}\left(x^{\prime}\right)\right)+L_{0}^{(2)} g\left(x^{\prime}\right), & \widetilde{W}_{3}\left(x^{\prime}\right)=0 & \text { in } \omega,
\end{array}
$$

where $K_{0}^{(k)}, L_{0}^{(k)} \in \mathbb{R}^{2 \times 2}, k=1,2$, are matrices with coefficients

$$
\left(K_{0}^{(k)}\right)_{i j}=\int_{Y} u_{j}^{i, k}(y) d y, \quad\left(L_{0}^{(k)}\right)_{i j}=\int_{Y} w_{j}^{i, k}(y) d y, \quad i, j=1,2,
$$

where $u^{i, k}, w^{i, k}$ are the solutions of the local micropolar problems defined in (5.68).

Here, $\tilde{P} \in H^{1}(\omega) \cap L_{0}^{2}(\omega)$ is the unique solution of the $2 D$ Darcy equation

$$
\left\{\begin{array}{l}
\operatorname{div}_{x^{\prime}}\left(K_{0}^{(1)}\left(f^{\prime}\left(x^{\prime}\right)-\nabla_{x^{\prime}} \tilde{P}\left(x^{\prime}\right)\right)+K_{0}^{(2)} g\left(x^{\prime}\right)\right)=0 \quad \text { in } \omega, \\
\left(K_{0}^{(1)}\left(f^{\prime}\left(x^{\prime}\right)-\nabla_{x^{\prime}} \tilde{P}\left(x^{\prime}\right)\right)+K_{0}^{(2)} g\left(x^{\prime}\right)\right) \cdot n=0 \quad \text { in } \partial \omega .
\end{array}\right.
$$

Proof. To eliminate the microscopic variable $y^{\prime}$ in the effective problem (5.65), we proceed as for the critical case by considering the local systems (5.68).

Thanks to the identities for the velocity $\int_{Y_{f}^{\prime}} \hat{U}\left(x^{\prime}, y^{\prime}\right) d y^{\prime}=\tilde{U}\left(x^{\prime}\right)$ with $\int_{Y_{f}^{\prime}} \hat{U}_{3} d y^{\prime}=0$ and the analogous one for the microrotation given in Lemma 5.1 we deduce that $\widetilde{U}$ and $\widetilde{W}$ are given by (5.69).

Finally, the divergence condition with respect to the variable $x^{\prime}$ given in (5.61) together with the expression of $\widetilde{U}^{\prime}\left(x^{\prime}\right)$ gives (5.70), which has a unique solution since $K_{0}^{(1)}$ is positive definite and then the whole sequence converges, see Part III - Theorem 2.5.2 in [17.

Remark 5.4. We observe that when $N$ is identically zero, taking into account the linear momentum equations from (5.65), we can deduce that the Darcy equation [5.70) agrees with the ones obtained in [5, 14] in the case HTPM. 


\section{The very thin porous medium (VTPM)}

It corresponds to the case when the cylinder height is much smaller than the interspatial distance, i.e. $a_{\varepsilon} \gg \varepsilon$ which is equivalent to $\lambda=+\infty$.

Next, we give some compactness results about the behavior of the extended sequences $\left(\tilde{u}_{\varepsilon}, \tilde{w}_{\varepsilon}, \tilde{P}_{\varepsilon}\right)$ and the unfolding functions $\left(\hat{u}_{\varepsilon}, \hat{w}_{\varepsilon}, \hat{P}_{\varepsilon}\right)$ satisfying the a priori estimates given in Lemmas 3.4 and 3.5 , and Lemma 3.7 . respectively.

Lemma 6.1. For a subsequence of $\varepsilon$ still denoted by $\varepsilon$, there exist the following functions:

i) (Velocity) there exist $\tilde{u} \in H_{0}^{1}\left(0,1 ; L^{2}(\omega)^{3}\right)$ with $\tilde{u}_{3}=0$ and $\hat{u} \in H_{0}^{1}\left(0,1 ; L_{\#}^{2}\left(\omega \times Y^{\prime}\right)^{3}\right)$ with $\hat{u}=0$ in $\omega \times Y_{s}$, such that $\int_{Y} \hat{u}\left(x^{\prime}, y\right) d y=\int_{0}^{1} \tilde{u}\left(x^{\prime}, y_{3}\right) d y_{3}$ with $\int_{Y} \hat{u}_{3} d y=0, \hat{u}_{3}$ independent of $y_{3}$ and moreover

$$
\begin{gathered}
\varepsilon^{-2} \tilde{u}_{\varepsilon} \rightarrow\left(\tilde{u}^{\prime}, 0\right) \text { in } H^{1}\left(0,1 ; L^{2}(\omega)^{3}\right), \quad \varepsilon^{-2} \hat{u}_{\varepsilon} \rightarrow \hat{u} \text { in } H^{1}\left(0,1 ; L^{2}\left(\omega \times Y^{\prime}\right)^{3}\right), \\
\operatorname{div}_{x^{\prime}}\left(\int_{0}^{1} \tilde{u}^{\prime}\left(x^{\prime}, y_{3}\right) d y_{3}\right)=0 \text { in } \omega, \quad\left(\int_{0}^{1} \tilde{u}^{\prime}\left(x^{\prime}, y_{3}\right) d y_{3}\right) \cdot n=0 \text { in } \partial \omega,
\end{gathered}
$$

$$
\operatorname{div}_{y^{\prime}} \hat{u}^{\prime}=0 \text { in } \omega \times Y_{f}, \quad \operatorname{div}_{x^{\prime}}\left(\int_{Y_{f}} \hat{u}^{\prime}\left(x^{\prime}, y\right) d y\right)=0 \text { in } \omega, \quad\left(\int_{Y_{f}} \hat{u}^{\prime}\left(x^{\prime}, y\right) d y\right) \cdot n=0 \text { in } \partial \omega,(6
$$

ii) (Microrotation) there exist $\tilde{w} \in H_{0}^{1}\left(0,1 ; L^{2}(\omega)^{3}\right)$ with $\tilde{w}_{3}=0$ and $\hat{w} \in H_{0}^{1}\left(0,1 ; L_{\#}^{2}\left(\omega \times Y^{\prime}\right)^{3}\right)$ with $\hat{w}=0$ in $\omega \times Y_{s}$, such that $\int_{Y_{f}} \hat{w}\left(x^{\prime}, y\right) d y=\int_{0}^{1} \tilde{w}\left(x^{\prime}, y_{3}\right) d y_{3}$ with $\int_{Y_{f}} \hat{w}_{3} d y=0, \hat{w}_{3}$ independent of $y_{3}$ and moreover

$$
\varepsilon^{-1} \tilde{w}_{\varepsilon} \rightarrow\left(\tilde{w}^{\prime}, 0\right) \text { in } H^{1}\left(0,1 ; L^{2}(\omega)^{3}\right), \quad \varepsilon^{-1} \hat{w}_{\varepsilon} \rightarrow \hat{w} \text { in } H^{1}\left(0,1 ; L^{2}\left(\omega \times Y^{\prime}\right)^{3}\right),
$$

iii) (Pressure) there exists $\tilde{P} \in L_{0}^{2}(\Omega)$ independent of $y_{3}$, such that

$$
\tilde{P}_{\varepsilon} \rightarrow \tilde{P} \text { in } L^{2}(\omega), \quad \hat{P}_{\varepsilon} \rightarrow \tilde{P} \text { in } L^{2}(\omega) .
$$

Proof. The proof of this result is obtained by arguing similarly to Section 5 in 4 and Lemma 4.1 of the present paper.

Theorem 6.2. In the case VTPM, the sequence $\left(\varepsilon^{-2} \hat{u}_{\varepsilon}, \varepsilon^{-1} \hat{w}_{\varepsilon}\right)$ converges weakly to $(\hat{u}, \hat{w})$ in $H^{1}\left(0,1 ; L^{2}(\omega \times\right.$ $\left.\left.Y^{\prime}\right)^{3}\right) \times H^{1}\left(0,1 ; L^{2}\left(\omega \times Y^{\prime}\right)^{3}\right)$ and $\hat{P}_{\varepsilon}$ converges strongly to $\tilde{P}$ in $L^{2}(\omega)$, where $(\hat{u}, \hat{w}, \tilde{P}) \in H_{0}^{1}\left(\omega ; L_{\#}^{2}\left(\omega \times Y^{\prime}\right)^{3}\right) \times$ $H_{0}^{1}\left(\omega ; L_{\#}^{2}\left(\omega \times Y^{\prime}\right)^{3}\right) \times\left(L_{0}^{2}(\omega) \cap H^{1}(\omega)\right)$ with $\hat{u}_{3}=\hat{w}_{3}=0$, is the unique solution of the following homogenized system

$$
\left\{\begin{aligned}
-\partial_{y_{3}} \hat{u}^{\prime}+\nabla_{y^{\prime}} \hat{q}=2 N^{2} \operatorname{rot}_{y_{3}} \hat{w}^{\prime}+f^{\prime}\left(x^{\prime}\right)-\nabla_{x^{\prime}} \tilde{P}\left(x^{\prime}\right) & \text { in } \omega \times Y_{f}, \\
-R_{c} \partial_{y_{3}} \hat{w}^{\prime}+4 N^{2} \hat{w}=2 N^{2} \operatorname{rot}_{y_{3}} \hat{u}^{\prime}+g^{\prime}\left(x^{\prime}\right) & \text { in } \omega \times Y_{f}, \\
\operatorname{div}_{y^{\prime}} \hat{u}^{\prime}=0 & \text { in } \omega \times Y_{f}, \\
\hat{u}^{\prime}=\hat{w}^{\prime}=0 & \text { in } \omega \times Y_{s}, \\
\operatorname{div}_{x^{\prime}}\left(\int_{Y} \hat{u}^{\prime}\left(x^{\prime}, y\right) d y\right)=0 & \text { in } \omega, \\
\left(\int_{Y} \hat{u}^{\prime}\left(x^{\prime}, y\right) d y\right) \cdot n=0 & \text { on } \partial \omega, \\
\hat{u}^{\prime}\left(x^{\prime}, y\right), \hat{w}^{\prime}\left(x^{\prime}, y\right), \hat{q}\left(x^{\prime}, y^{\prime}\right) \quad Y^{\prime}-\text { periodic. } &
\end{aligned}\right.
$$


Proof. We choose $\varphi \in \mathcal{D}\left(\omega ; C_{\#}^{\infty}(Y)^{3}\right)$ with $\operatorname{div}_{y^{\prime}} \varphi^{\prime}=0$ in $\omega \times Y, \operatorname{div}_{x^{\prime}}\left(\int_{Y} \varphi^{\prime} d y\right)=0$ in $\omega$ and $\varphi_{3}$ independent of $y_{3}$ in (3.43). Taking into account that thanks to $\operatorname{div}_{y^{\prime}} \varphi^{\prime}=0$ in $\omega \times Y$ and $\varphi_{3}$ independent of $y_{3}$, we have that

$$
\frac{1}{a_{\varepsilon}} \int_{\omega \times Y} \hat{P}_{\varepsilon} \operatorname{div}_{y^{\prime}} \varphi^{\prime} d x^{\prime} d y=0 \text { and } \frac{1}{\varepsilon} \int_{\omega \times Y} \partial_{y_{3}} \hat{P}_{\varepsilon} \partial_{y_{3}} \varphi_{3} d x^{\prime} d y=0 .
$$

Thus, passing to the limit using the convergences (6.71), 6.74), 6.75), $\varepsilon / a_{\varepsilon} \rightarrow 0$ and using in the limit that $\tilde{P}$ does not depend on $y$ and $\operatorname{div}_{x^{\prime}}\left(\int_{Y} \varphi^{\prime} d y\right)=0$, we obtain

$$
\left\{\begin{array}{l}
\int_{\omega \times Y} D_{y^{\prime}} \hat{u}^{\prime}: D_{y^{\prime}} \varphi^{\prime} d x^{\prime} d y=2 N^{2} \int_{\omega \times Y} \operatorname{rot}_{y_{3}} \hat{w}^{\prime} \cdot \varphi^{\prime} d x^{\prime} d y+\int_{\omega \times Y} f^{\prime} \cdot \varphi^{\prime} d x^{\prime} d y, \\
\int_{\omega \times Y} \nabla_{y^{\prime}} \hat{u}_{3}: \nabla_{y^{\prime}} \varphi_{3} d x^{\prime} d y=0 .
\end{array}\right.
$$

Next, for every $\psi \in \mathcal{D}\left(\omega ; C_{\#}^{\infty}(Y)^{3}\right)$ with $\psi_{3}$ independent of $y_{3}$, we choose $\psi_{\varepsilon}=a_{\varepsilon}^{-1} \psi$ in (3.44) with $g_{\varepsilon}$ and $R_{M}$ satisfying (3.16) and (3.17). Then, passing to the limit using convergences (6.71) and (6.74), we get

$$
\left\{\begin{array}{l}
R_{c} \int_{\omega \times Y_{f}} \partial_{y_{3}} \hat{w}^{\prime}: \partial_{y_{3}} \psi^{\prime} d x^{\prime} d y+4 N^{2} \int_{\omega \times Y_{f}} \hat{w}^{\prime} \cdot \psi^{\prime} d x^{\prime} d y=2 N^{2} \int_{\omega \times Y_{f}} \operatorname{rot}_{y_{3}} \hat{u}^{\prime} \cdot \psi^{\prime} d x^{\prime} d y+\int_{\omega \times Y_{f}} g^{\prime}\left(x^{\prime}\right) \cdot \psi^{\prime} d x^{\prime} d y \\
R_{c} \int_{\omega \times Y_{f}} \partial_{y_{3}} \hat{w}_{3}: \partial_{y_{3}} \psi_{3} d x^{\prime} d y+4 N^{2} \int_{\omega \times Y_{f}} \hat{w}_{3} \cdot \psi_{3} d x^{\prime} d y=0 .
\end{array}\right.
$$

The second equations of (6.77) and (6.78) together to the boundary conditions imply $\hat{u}_{3}=\hat{w}_{3}=0$. By density, we can deduce that this variational formulation is equivalent to problem (6.76).

Let us define the local problems which are useful to eliminate the variable $y$ of the previous homogenized problem and then obtain a Darcy equation for $\tilde{P}$. We define $\Phi$ and $\Psi$ by

$$
\begin{gathered}
\Phi\left(N, R_{c}\right)=\frac{1}{12}+\frac{R_{c}}{4\left(1-N^{2}\right)}-\frac{1}{4} \sqrt{\frac{N^{2} R_{c}}{1-N^{2}}} \operatorname{coth}\left(N \sqrt{\frac{1-N^{2}}{R_{c}}}\right), \\
\Psi\left(N, R_{c}\right)=\frac{\tanh \left(N \sqrt{\frac{1-N^{2}}{R_{c}}}\right)}{1-N \sqrt{\frac{1-N^{2}}{R_{c}}} \tanh \left(N \sqrt{\frac{1-N^{2}}{R_{c}}}\right)},
\end{gathered}
$$

and for every $k=1,2$, we consider the following 2D local micropolar Darcy problems for $\pi^{i, k}$ as follows

$$
\left\{\begin{aligned}
-\operatorname{div}_{y^{\prime}}\left(\frac{1}{1-N^{2}} \Phi\left(N, R_{c}\right)\left(\nabla_{y^{\prime}} \pi^{i, k}\left(y^{\prime}\right)+e_{i} \delta_{1 k}\right)\right)=0 & \text { in } Y_{f}^{\prime} \\
\left(\frac{1}{1-N^{2}} \Phi\left(N, R_{c}\right)\left(\nabla_{y^{\prime}} \pi^{i, k}\left(y^{\prime}\right)+e_{i} \delta_{1 k}\right)\right) \cdot n=0 & \text { in } \partial Y_{s}^{\prime} .
\end{aligned}\right.
$$

It is known that from the positivity of function $\Phi$, problem (6.85) has a unique solution for $\pi^{i, k} \in H_{\#}^{1}\left(Y^{\prime}\right)$ (see 7] for more details).

Theorem 6.3. Let $(\hat{u}, \hat{w}, \tilde{P}) \in H_{0}^{1}\left(\omega ; L_{\#}^{2}\left(\omega \times Y^{\prime}\right)^{3}\right) \times H_{0}^{1}\left(\omega ; L_{\#}^{2}\left(\omega \times Y^{\prime}\right)^{3}\right) \times\left(L_{0}^{2}(\omega) \cap H^{1}(\omega)\right)$ be the unique weak solution of problem (6.76). Then, the extensions $\left(\varepsilon^{-2} \tilde{u}_{\varepsilon}, \varepsilon^{-1} \tilde{w}_{\varepsilon}\right)$ and $\tilde{P}_{\varepsilon}$ of the solution of problem (2.10)- (2.11) converge weakly to $(\tilde{u}, \tilde{w})$ in $H^{1}\left(0,1 ; L^{2}(\omega)^{3}\right) \times H^{1}\left(0,1 ; L^{2}(\omega)^{3}\right)$ and strongly to $\tilde{P}$ in $L^{2}(\omega)$ respectively, with $\tilde{u}_{3}=\tilde{w}_{3}=0$. Moreover, defining $\widetilde{U}\left(x^{\prime}\right)=\int_{0}^{1} \tilde{u}\left(x^{\prime}, y_{3}\right) d y_{3}$ and $\widetilde{W}\left(x^{\prime}\right)=\int_{0}^{1} \tilde{w}\left(x^{\prime}, y_{3}\right) d y_{3}$, it holds

$$
\begin{array}{lll}
\widetilde{U}^{\prime}\left(x^{\prime}\right)=K_{\infty}^{(1)}\left(f^{\prime}\left(x^{\prime}\right)-\nabla_{x^{\prime}} \tilde{P}\left(x^{\prime}\right)\right)+K_{\infty}^{(2)} g\left(x^{\prime}\right), & \widetilde{U}_{3}\left(x^{\prime}\right)=0 & \text { in } \omega, \\
\widetilde{W}^{\prime}\left(x^{\prime}\right)=L_{\infty}^{(2)} g\left(x^{\prime}\right), & \widetilde{W}_{3}\left(x^{\prime}\right)=0 & \text { in } \omega,
\end{array}
$$


where the matrices $K_{\infty}^{(k)} \in \mathbb{R}^{2 \times 2}, k=1,2$, and $L_{\infty}^{(2)} \in \mathbb{R}^{2 \times 2}$ are matrices with coefficients

$$
\begin{aligned}
& \left(K_{\infty}^{(k)}\right)_{i j}=\frac{1}{1-N^{2}} \int_{Y^{\prime}} \Phi\left(N, R_{c}\right)\left(\partial_{y_{i}} \pi^{j, k}\left(y^{\prime}\right)+\delta_{i j} \delta_{1 k}\right) d y^{\prime}, \quad i, j=1,2, \\
& \left(L_{\infty}^{(2)}\right)_{i j}=-\frac{1}{4 N^{3}} \sqrt{\frac{R_{c}}{1-N^{2}}}\left(\int_{Y^{\prime}} \Psi\left(N, R_{c}\right) d y^{\prime}\right) \delta_{i j},
\end{aligned}
$$

with $\Phi$ and $\Psi$ given by (6.79) and (6.80) respectively, and $\pi^{i, k} \in H_{\#}^{1}\left(Y^{\prime}\right), i, k=1,2$, the unique solutions of the local problems (6.85). Here, $\tilde{P} \in H^{1}(\omega) \cap L_{0}^{2}(\omega)$ is the unique solution of the $2 D$ Darcy problem

$$
\left\{\begin{array}{l}
\operatorname{div}_{x^{\prime}}\left(K_{\infty}^{(1)}\left(f^{\prime}\left(x^{\prime}\right)-\nabla_{x^{\prime}} \tilde{P}\left(x^{\prime}\right)\right)+K_{\infty}^{(2)} g\left(x^{\prime}\right)\right)=0 \quad \text { in } \omega, \\
\left(K_{\infty}^{(1)}\left(f^{\prime}\left(x^{\prime}\right)-\nabla_{x^{\prime}} \tilde{P}\left(x^{\prime}\right)\right)+K_{\infty}^{(2)} g\left(x^{\prime}\right)\right) \cdot n=0 \quad \text { in } \partial \omega .
\end{array}\right.
$$

Proof. We proceed as in in the proof of Theorem 4.3 in order to obtain (6.82). Thus, by using (4.59) where $\left(u^{i, k}, w^{i, k}, \pi^{i, k}\right) \in H_{0, \#}^{1}\left(Y_{f}\right)^{2} \times H_{0, \#}^{1}\left(Y_{f}\right)^{2} \times L_{0}^{2}\left(Y_{f}^{\prime}\right), i, k=1,2$, is the unique solution of

$$
\left\{\begin{array}{rc}
-\partial_{y_{3}} u^{i, k}+\nabla_{y^{\prime}} \pi^{i, k}-2 N^{2} \operatorname{rot}_{y_{3}} w^{i, k}=-e_{i} \delta_{1 k} & \text { in } Y_{f}, \\
\operatorname{div}_{y^{\prime}} u^{i, k}=0 & \text { in } Y_{f}, \\
-R_{c} \partial_{y_{3}} w^{i, k}+4 N^{2} w^{i, k}-2 N^{2} \operatorname{rot}_{y_{3}} u^{i, k}=-e_{i} \delta_{2 k} & \text { in } Y_{f}, \\
u^{i, k}=w^{i, k}=0 & \text { in } Y_{s}, \\
\int_{Y^{\prime}} u_{3}^{i, k}\left(y^{\prime}\right) d y^{\prime}=\int_{Y^{\prime}} w_{3}^{i, k}\left(y^{\prime}\right) d y^{\prime}=0, & \\
u^{i, k}(y), w^{i, k}(y), \pi^{i, k}\left(y^{\prime}\right) & Y^{\prime} \text { - periodic, }
\end{array}\right.
$$

then, thanks to the identities $\int_{Y_{f}} \hat{u}\left(x^{\prime}, y\right) d y=\int_{0}^{1} \tilde{u}\left(x^{\prime}, y_{3}\right) d y_{3}$ with $\hat{u}_{3}=0$ and $\int_{Y_{f}} \hat{w}\left(x^{\prime}, y\right) d y=\int_{0}^{1} \tilde{w}\left(x^{\prime}, y_{3}\right) d y_{3}$ with $\hat{w}_{3}=0$ given in Lemma 6.1 it holds

$$
\begin{aligned}
& \widetilde{U}^{\prime}\left(x^{\prime}\right)=\int_{Y} \hat{u}^{\prime}\left(x^{\prime}, y\right) d y=-K_{\infty}^{(1)}\left(\nabla_{x^{\prime}} \tilde{P}\left(x^{\prime}\right)-f^{\prime}\left(x^{\prime}\right)\right)+K_{\infty}^{(2)} g^{\prime}\left(x^{\prime}\right), \quad \widetilde{U}_{3}\left(x^{\prime}\right)=\int_{Y} \hat{u}_{3}\left(x^{\prime}, y^{\prime}\right) d y=0 \quad \text { in } \omega, \\
& \widetilde{W}^{\prime}\left(x^{\prime}\right)=\int_{Y} \hat{w}^{\prime}\left(x^{\prime}, y\right) d y=-L_{\infty}^{(1)}\left(\nabla_{x^{\prime}} \tilde{P}\left(x^{\prime}\right)-f^{\prime}\left(x^{\prime}\right)\right)+L_{\infty}^{(2)} g^{\prime}\left(x^{\prime}\right), \quad \widetilde{W}_{3}\left(x^{\prime}\right)=\int_{Y} \hat{w}_{3}\left(x^{\prime}, y\right) d y=0 \quad \text { in } \omega,
\end{aligned}
$$

where $K_{\infty}^{(k)}, L_{\infty}^{(k)}, k=1,2$, are matrices defined by their coefficients

$$
\left(K_{\infty}^{(k)}\right)_{i j}=-\int_{Y} u_{j}^{i, k}(y) d y, \quad\left(L_{\infty}^{(k)}\right)_{i j}=-\int_{Y} w_{j}^{i, k}(y) d y, \quad i, j=1,2 .
$$

Then, by the divergence condition in the variable $x^{\prime}$ given in (6.76), we get the Darcy equation (6.84).

However, we observe that (6.85) can be viewed as a system of ordinary differential equations with constant coefficients, with respect to the variable $y_{3}$ and unkowns functions $y_{3} \mapsto u_{1}^{i, k}\left(y^{\prime}, y_{3}\right), w_{2}^{i, k}\left(y^{\prime}, y_{3}\right), u_{2}^{i, k}\left(y^{\prime}, y_{3}\right)$, $w_{1}^{i, k}\left(y^{\prime}, y_{3}\right)$, where $y^{\prime}$ is a parameter, $y^{\prime} \in Y^{\prime}$. Thus, we can give explicit expressions for $u^{i, k}$ and $w^{i, k}$ given in terms of $\pi^{i, k}$ as follows (see [7] and [8] for more details):

$$
\begin{aligned}
u^{i, k}(y)= & \frac{1}{2\left(1-N^{2}\right)}\left[y_{3}^{2}-y_{3}+\frac{N^{2}}{k}\left(\sinh \left(k y_{3}\right)-\left(\cosh \left(k y_{3}\right)-1\right) \operatorname{coth}\left(\frac{k}{2}\right)\right)\right]\left(\nabla_{y^{\prime}} \pi^{i, k}\left(y^{\prime}\right)+e_{i} \delta_{1 k}\right) \\
& +\frac{1}{N^{2}}\left[\left(\frac{2 N^{2}}{k} \sinh \left(k y_{3}\right)-2 y_{3}\right) A+\frac{2 N^{2}}{k}\left(\cosh \left(k y_{3}\right)-1\right) B-y_{3}\right]\left(e_{i} \delta_{2 k}\right)^{\perp}, \\
w^{i, k}(y)= & \frac{1}{4\left(1-N^{2}\right)}\left[2 y_{3}+\left(\cosh \left(k y_{3}\right)-1-\sinh \left(k y_{3}\right) \operatorname{coth}\left(\frac{k}{2}\right)\right)\right]\left(\nabla_{y^{\prime}} \pi^{i, k}\left(y^{\prime}\right)+e_{i} \delta_{1 k}\right)^{\perp} \\
& -\frac{1}{2 N^{2}}\left[\cosh \left(k y_{3}\right) A+\sinh \left(k y_{3}\right) B\right] e_{i} \delta_{2 k},
\end{aligned}
$$


where $k=\sqrt{\frac{4 N^{2}\left(1-N^{2}\right)}{R_{c}}}$ and $A, B$ are given by

$$
A\left(y^{\prime}\right)=\frac{\sinh (k)}{-2 \sinh (k)+\frac{4 N^{2}}{k}(\cosh (k)-1)}, \quad B\left(y^{\prime}\right)=\frac{-(\cosh (k)-1)}{-2 \sinh (k)+\frac{4 N^{2}}{k}(\cosh (k)-1)} .
$$

Integrating with respect to $y_{3}$, we get

$$
\begin{aligned}
& \int_{0}^{1} u^{i, k}\left(y^{\prime}, y_{3}\right) d y_{3}=-\frac{1}{1-N^{2}} \Phi\left(N, R_{c}\right)\left(\nabla_{y^{\prime}} \pi^{i, k}\left(y^{\prime}\right)+e_{i} \delta_{1 k}\right) \\
& \int_{0}^{1} w^{i, k}\left(y^{\prime}, y_{3}\right) d y_{3}=\frac{1}{4 N^{3}} \sqrt{\frac{R_{c}}{1-N^{2}}} \Psi\left(N, R_{c}\right) e_{i} \delta_{2 k}
\end{aligned}
$$

with $\Phi$ and $\Psi$ given by (6.79) and (6.80), and so that $\pi^{i, k}$ satisfies the Darcy local problem (6.81). Using the expressions of $u^{i, k}$ and $w^{i, k}$ together with (6.86), (6.87) and (6.89), we easily get (6.82), which has a unique solution since $K_{\infty}^{(1)}$ is positive definite and then the whole sequence converges. Observe that, from the second equation in (6.89) with $k=2$, we have $L_{0}^{(1)}=0$, which ends the proof.

Remark 6.4. We observe that then $R_{c}$ tends to zero, the function $\Phi$ given by 6.79 becomes identical to $1 / 12$. In that case, when $N$ is identically zero, taking into account the linear momentum equations from 6.76), we can deduce that the Darcy equation [6.84) agrees with the ones obtained in [5, 14] in the case VTPM.

\section{References}

[1] G. Allaire, Homogenization of the Stokes flow in a connected porous medium, Asymptotic Analysis, 2 (1989) $203-222$.

[2] M. Anguiano and R. Bunoiu, On the flow of a viscoplastic fluid in a thin periodic domain. Constanda C. , Harris P. (eds) Integral Methods in Science and Engineering, Birkäuser, Cham., (2019).

[3] M. Anguiano and R. Bunoiu, Homogenization of Bingham flow in thin porous media, Networks and Heterogeneous Media, 15 (2020).

[4] M. Anguiano and F.J. Suárez-Grau, Homogenization of an incompressible non-Newtonian flow through a thin porous medium, ZAMP-Journal of Applied Mathematics and Physics, (2017) 68:45, DOI: 10.1007/s00033017-0790-z.

[5] M. Anguiano and F.J. Suárez-Grau, The transition between the Navier-Stokes equations to the Darcy equation in a thin porous medium, Mediterr. J. Math., (2018) 15:45.

[6] T. Arbogast, J. Douglas J.R. and U. Hornung, Derivation of the double porosity model of single phase flow via homogenization theory, SIAM J. Math. Anal., 21 (1990) 823-836.

[7] G. Bayada, M. Chambat and S.R. Gamouana, About thin film micropolar asymptotic equations, Quart. Appl. Math., 59 (2001) 413-439.

[8] G. Bayada and G. Lukaszewicz, On micropolar fluids in the theory of lubrication. Rigorous derivation of an analogue of the Reynolds equation. Internat. J. Engrg. Sci., 34 (1996) 1477-1490.

[9] D. Cioranescu, A. Damlamian and G. Griso, Periodic unfolding and homogenization, C.R. Acad. Sci. Paris Ser. I, 335 (2002) 99-104.

[10] D. Cioranescu, A. Damlamian and G. Griso, The periodic unfolding method in homogenization, SIAM J. Math. Anal., 40 (2008) 1585-1620. 
[11] G. Duvaut and J.L. Lions, Les inequations en mechanique et en physique [The inequations in mechanics and physics], Dunod, Paris (1972).

[12] A.C. Eringen, Simple mocrofluids, Internat. J. Engrg. Sci., 2 (1964) 205-207.

[13] A.C. Eringen, Theory of micropolar fluids, J. Math. Mech., 16 (1966) 1-18.

[14] J. Fabricius, J. Gunnar I. Hellström, T. Staffan Lundström, Elena Miroshnikova and Peter Wall, Darcy's Law for flow in a periodic thin porous medium confined between two parallel plates, Transp. Porous Med., 115 (2016) 473-493.

[15] V. Frishfelds, T.S. Lundström and A. Jakovics, Lattice gas analysis of liquid front in non-crimp fabrics, Transp. Porous Med., 84 (2011) 75-93

[16] W. Jeon and C.B. Shin, Design and simulation of passive mixing in microfluidic systems with geometric variations, Chem. Eng. J., 152 (2009) 575-582.

[17] G. Lukaszewicz, Micropolar fluids, theory and applications, Modeling and Simulation in Science, Engineering and Technology, Birkhaüser, (1999).

[18] T.S. Lundström, S. Toll, and J.M. Hakanson, Measurements of the permeability tensor of compressed fibre beds, Transp. Porous Med., 47 (2002) 363-380.

[19] M. Nordlund and T.S. Lundström, Effect of multi-scale porosity in local permeability modelling of non-crimp fabrics, Transp. Porous Med., 73 (2008) 109-124.

[20] F. Singh, B. Stoeber, and S.I. Green, Micro-PIV measurement of flow upstream of papermaking forming fabrics, Transp. Porous Med., 107 (2015) 435-448.

[21] H. Tan and K.M. Pillai, Multiscale modeling of unsaturated flow in dual-scale fiber preforms of liquid composite molding I: Isothermal flows, Compos. Part A Appl. Sci. Manuf., 43 (2012) 1-13.

[22] L. Tartar, Incompressible fluid flow in a porous medium convergence of the homogenization process, Appendix to Lecture Notes in Physics, 127, Berlin, Springer-Velag, 1980. 